\title{
Silician Magnetite: Si-Fe-Nanoprecipitates and Other Mineral Inclusions in Magnetite from the Olympic Dam Deposit, South Australia
}

\author{
Cristiana L. Ciobanu ${ }^{1, *}$, Max R. Verdugo-Ihl ${ }^{1}$, Ashley Slattery ${ }^{2}$, Nigel J. Cook ${ }^{1}{ }^{\mathbb{D}}$, \\ Kathy Ehrig ${ }^{3}(\mathbb{D})$, Liam Courtney-Davies ${ }^{1}(\mathbb{D})$ and Benjamin P. Wade ${ }^{2}$ \\ 1 School of Chemical Engineering, The University of Adelaide, Adelaide, SA 5005, Australia; \\ max.verdugoihl@adelaide.edu.au (M.R.V.-I.); nigel.cook@adelaide.edu.au (N.J.C.); \\ liam.courtney-davies@adelaide.edu.au (L.C.-D.) \\ 2 Adelaide Microscopy, The University of Adelaide, Adelaide, SA 5005, Australia; \\ ashley.slattery@adelaide.edu.au (A.S.); benjamin.wade@adelaide.edu.au (B.P.W.) \\ 3 BHP Olympic Dam, Adelaide, SA 5000, Australia; kathy.ehrig@bhp.com \\ * Correspondence: cristiana.ciobanu@adelaide.edu.au; Tel.: +61-432-955-000
}

Received: 19 April 2019; Accepted: 16 May 2019; Published: 20 May 2019

\begin{abstract}
A comprehensive nanoscale study on magnetite from samples from the outer, weakly mineralized shell at Olympic Dam, South Australia, has been undertaken using atom-scale resolution High Angle Annular Dark Field Scanning Transmission Electron Microscopy (HAADF STEM) imaging and STEM energy-dispersive X-ray spectrometry mapping and spot analysis, supported by STEM simulations. Silician magnetite within these samples is characterized and the significance of nanoscale inclusions in hydrothermal and magmatic magnetite addressed. Silician magnetite, here containing $\mathrm{Si}-\mathrm{Fe}$-nanoprecipitates and a diverse range of nanomineral inclusions [(ferro)actinolite, diopside and epidote but also U-, W-(Mo), Y-As- and As-S-nanoparticles] appears typical for these samples. We observe both silician magnetite nanoprecipitates with spinel-type structures and a $\gamma-\mathrm{Fe}_{1.5} \mathrm{SiO}_{4}$ phase with maghemite structure. These are distinct from one another and occur as bleb-like and nm-wide strips along $d_{111}$ in magnetite, respectively. Overprinting of silician magnetite during transition from K-feldspar to sericite is also expressed as abundant lattice-scale defects (twinning, faults) associated with the transformation of nanoprecipitates with spinel structure into maghemite via Fe-vacancy ordering. Such mineral associations are characteristic of early, alkali-calcic alteration in the iron-oxide copper gold (IOCG) system at Olympic Dam. Magmatic magnetite from granite hosting the deposit is quite distinct from silician magnetite and features nanomineral associations of hercynite-ulvöspinel-ilmenite. Silician magnetite has petrogenetic value in defining stages of ore deposit evolution at Olympic Dam and for IOCG systems elsewhere. The new data also add new perspectives into the definition of silician magnetite and its occurrence in ore deposits.
\end{abstract}

Keywords: silician magnetite; high angle annular dark field scanning transmission electron microscopy; Olympic Dam

\section{Introduction}

Magnetite $\left(\mathrm{Fe}^{2+} \mathrm{Fe}^{3+}{ }_{2} \mathrm{O}_{4}\right)$, an abundant mineral in ore deposits of various genetic types, is one of the ' $2-3$ oxide spinels', $\mathrm{A}^{2+} \mathrm{B}^{3+}{ }_{2} \mathrm{O}_{4}$; Table 1 ([1] and references therein). The oxide spinel structure has a cubic close packing of anions $(\mathrm{O})$, with the cations hosted either in tetrahedral $(\mathrm{T})$ or octahedral (M) sites (e.g., [2]). Displacement of metal ions along the [111] direction leads to a lowering of $F d \overline{3} m$ symmetry, for example $F \overline{4} 3 m$ symmetry was reported in natural magnetite ([3]; Table 1). Cation disorder 
over $\mathrm{T}$ and $\mathrm{M}$ sites expressed as parameter $i$ can vary from $i=0$ in normal spinels, ${ }^{\mathrm{T}} \mathrm{A}^{\mathrm{M}} \mathrm{B}_{2} \mathrm{O}_{4}$, to $i$ $=1$ in inverse spinels, ${ }^{\mathrm{T}} \mathrm{B}^{\mathrm{M}}(\mathrm{AB}) \mathrm{O}_{4}$, such as magnetite. Of particular petrogenetic significance are the magnetite-ulvöspinel and magnetite-ahrensite solid solution series. These are two examples of paired "2-3" and "4-2" spinel series that involve coupled substitutions between A and B cations to accommodate the charge balance in the $\mathrm{T}$ and $\mathrm{M}$ sites. Electron hopping (exchange of $\mathrm{Fe}^{2+}$ and $\mathrm{Fe}^{3+}$ in the M sites; [4]), or the semi-conductor character (defect structure of natural magnetite at room temperature; [5]) of magnetite introduces further complexity in evaluating the ordering, full/partial occupancy of the $\mathrm{T}$ and $\mathrm{M}$ sites in these two solid solution series.

In natural samples, the Fe-Ti-oxides form complex intergrowths with one another as a response to exsolution from high-T solid solutions [6]. Relationships among Fe-Ti-oxides within the $\mathrm{FeO}-\mathrm{TiO}_{2}-\mathrm{Fe}_{2} \mathrm{O}_{3}$ compositional triangle are represented by tie-lines between (i) magnetite and ulvöspinel $\left(\mathrm{TiFe}_{2} \mathrm{O}_{4}\right)$, and (ii) ilmenite $\left(\mathrm{FeTiO}_{3}\right)$ and hematite/maghemite, which define the titanomagnetite and hemoilmenite solid solution series with cubic and rhombohedral lattices, respectively ([7], and references therein). In igneous rocks, the Fe-Ti-oxides occur as accessory minerals that crystallize at $\mathrm{T}>600{ }^{\circ} \mathrm{C}$, leading to titaniferous magnetite via incorporation of $\mathrm{Ti}^{4+}$ into magnetite (ulvöspinel component). Upon cooling, sub-solidus re-equilibration among Fe-Ti-oxides results in crystallographically-oriented intergrowths between magnetite and ilmenite (trellis textures), which have been interpreted either in the context of an "oxidation-exsolution" model [8] or a reduction $\mathrm{O}_{2}$-conserving scheme $[9,10]$. Although these models are widely used to interpret cooling histories among Fe-Ti-oxides, and carry implications for ore-forming processes, they cannot completely explain the complexity of intergrowths between magnetite, ilmenite and ulvöspinel observed in natural rocks (e.g., [11]). Furthermore, nanoscale studies show sub-sets of intergrowths between ilmenite and non-titaniferous spinels (e.g., hercynite) in titanomagnetite from layered intrusions [12].

The silicate spinel ringwoodite and its natural Fe-analogue, ahrensite (Table 1) are the high-pressure polymorphs of minerals from the olivine group. They are of petrological interest because of their role in understanding the Earth's mantle in the transitional zone at depths between 400 and $650 \mathrm{~km}$ ([1], and references therein). The single natural occurrence of ahrensite is from a reaction rim on olivine at the contact with shock-induced melt pockets produced in the Tissint meteorite [13].

An average of $1.2 \mathrm{wt}$. \% Si (equivalent to $9 \mathrm{~mol} \% \mathrm{Fe}_{2} \mathrm{SiO}_{4}$ ) was measured in titanomagnetite occurring as a microphenocryst in an ash flow [14]. This was one of the first studies to address the presence of Si in magnetite at the nanoscale using Transmission Electron Microscopy (TEM) with composition measured by both electron microprobe and energy-dispersive spectroscopy (EDS) Scanning TEM (STEM). The sample displayed dense magnetite-ilmenite intergrowths and Si was found to be present as very small silicate inclusions but also as unusual Si-rich domains of uncertain origin in magnetite and to some extent also in ilmenite. Newberry et al. [14] acknowledge that the inclusions and domains may be irregularly distributed throughout the magnetite and have sizes well below those resolvable with the electron microprobe. The Si content was attributed to solid solution in the spinel structure with 'fayalite-type substitution' $\left(2 \mathrm{Fe}^{3+}=\mathrm{Si}^{4+}+\mathrm{Fe}^{2+}\right)$.

The complete compositional range between $\mathrm{Fe}_{2} \mathrm{SiO}_{4}$ and $\mathrm{Fe}_{3} \mathrm{O}_{4}$ spinel solid solution was assessed at $9 \mathrm{GPa}$ and $1200{ }^{\circ} \mathrm{C}$ [15]. In a study of electric conductivity relative to non-stoichiometry, discrete intermediate species, $\mathrm{Fe}_{1-\mathrm{x}} \mathrm{Si}_{\mathrm{x}} \mathrm{O}_{4}(0<\mathrm{x}<0.288$; Table 1), were synthesized at 6-10 GPa and $1200{ }^{\circ} \mathrm{C}$. These were subsequently characterized by X-Ray powder diffraction as spinel single phase structures with composition calculated from microprobe analysis [16]. The cation distribution/deficiency relating to non-stoichiometry was discussed with respect to electron hopping with the conclusion that the electrical conductivity is affected by the Si content $(x)$ and hopping activation energy in the $M$ site, an important finding for geophysical studies. A disorder parameter $(t)$ with non-linear variation of lattice constants with composition was postulated and a general crystal-chemical formula for the studied spinel solid solution was given as:

$$
{ }^{\mathrm{T}}\left[\mathrm{Fe}^{3+}{ }_{1-x+x t}, \mathrm{Si}^{4+}{ }_{x(1-t)}\right]^{\mathrm{M}}\left[\mathrm{Fe}^{2+}{ }_{1+x}, \mathrm{Fe}^{3+}{ }_{1-x-x t}, \mathrm{Si}^{4+}{ }_{x t}\right] \mathrm{O}_{4}
$$


Although many studies from the 1970's report "silica-bearing magnetite" (e.g., [17]), the term "silician magnetite" was attributed to Shiga [18], who refers to magnetite with $>1 \mathrm{wt}$. $\% \mathrm{SiO}_{2}$ and depleted in other cations except ferrous and ferric ions in samples from the Kamaishi $\mathrm{Cu}-\mathrm{Fe}$ skarn, Japan. Magnetite with various contents of $\mathrm{SiO}_{2}$ (up to $\sim 9$ wt. $\% \mathrm{SiO}_{2}$ ) has been documented in deposits spanning the magmatic-hydrothermal spectrum, i.e., from banded iron formation (BIF) ores metamorphosed at low conditions, as well as in mafic and ultramafic rocks (see summary and references given in Huberty et al. [19]). In an overview study by Shimazaki [20], the presence of additional components in Si-bearing magnetite, notably $\mathrm{Al}_{2} \mathrm{O}_{3}, \mathrm{CaO}$, and $\mathrm{MgO}$, was considered as either being incorporated in the magnetite or due to exsolution of silicates, but distinct from "silician magnetite" sensu stricto. The author argues that, besides 'spinel' solid solution, there exist mechanisms of $\mathrm{Si}$ incorporation with vacancies leading to structures kin to maghemite. The latter is known for a variety of crystal structures with disordered to full-site vacancy ordering (Table 1; [21] and references therein).

Table 1. Overview of minerals and phases discussed.

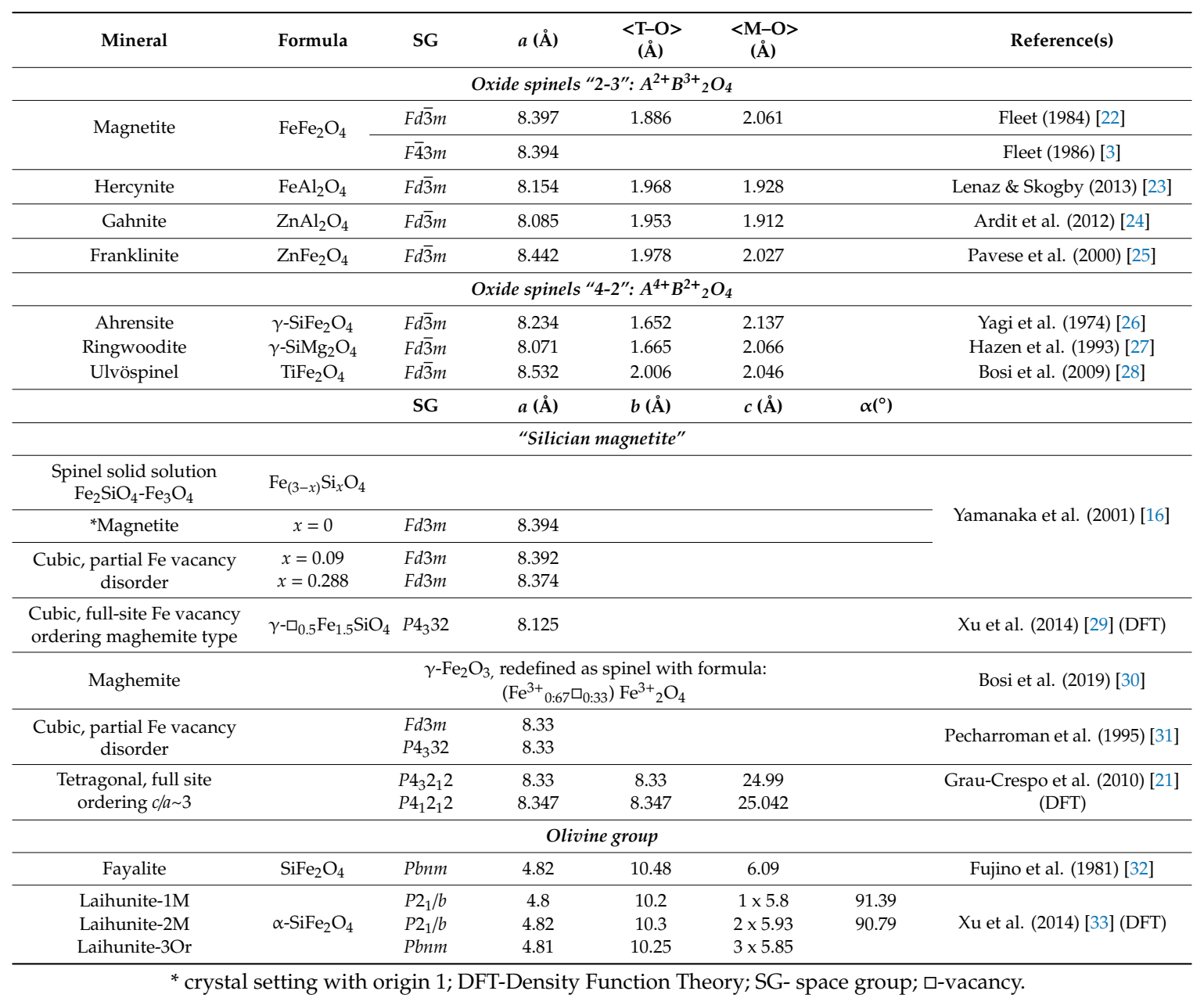

Silician magnetite with 1-3 wt. \% $\mathrm{SiO}_{2}$ content is reported from metamorphosed BIFs from the Proterozoic Hamersley Group, Western Australia [19]. A large electron probe microanalysis (EPMA) dataset is fit to a trend inferring the presence of maghemite-type vacancies. This fit is supported by nanoscale study of the same BIF material. Identification and definition of Si-magnetite nanoprecipitates as discrete phases with ordered maghemite-type vacancies was only achievable by using Z-contrast techniques such as High Angle Annular Dark Field (HAADF) STEM imaging at atom-scale resolution [29]. In combination with density functional theory (DFT) methods, the authors defined both the composition and crystal structure of the discrete Fe-Si-phase (Table 1). The structural 
model shows $\mathrm{Si}^{4+}$ cations replacing $\mathrm{Fe}^{3+}$ in $\mathrm{T}$ sites whereas vacancies are introduced in the $\mathrm{M}$ sites. The Si-magnetite reported by $\mathrm{Xu}$ et al. [29] occurs as thin bands along $\{111\}$ directions in magnetite with widths of even multiples of $d_{111}$. The olivine group is a further example of nanomineral phase identification using Z-contrast imaging and ab initio DFT modelling [33]. The same authors have shown that, aside from fayalite, a polymorph of ahrensite (Table 1), several intermediate phases featuring vacancy ordering and superstructuring can co-exist with one another at the nanoscale (Table 1).

Based on data available at the time, Newberry et al. [14] stipulates that higher $\mathrm{SiO}_{2}$ (up to 2-3 wt. \%) should be present in magnetite from high-level granites and rhyolites relative to basalts, kimberlites and metamorphic magnetites ( $<0.5 \mathrm{wt} . \% \mathrm{SiO}_{2}$ ). The empirical correlation between $\mathrm{Si}$ with $\mathrm{Ca}$ and $\mathrm{Mg}$ was interpreted as substitution of $\mathrm{Ca}_{2} \mathrm{SiO}_{4}$ and $\mathrm{Mg}_{2} \mathrm{SiO}_{4}$ in magnetite, or presence of sub-micron silicate inclusions thereof. Minor to trace amounts of other elements ( $\mathrm{Mg}, \mathrm{Zn}, \mathrm{Al}, \mathrm{Ti}, \mathrm{Mn}$ ) are detected even in those BIF samples from which Si-magnetite nanoprecipitates were identified [19,29]. In such a case, silician magnetite (1-3 wt. \% $\left.\mathrm{SiO}_{2}\right)$ forms various textures with $\mathrm{Si}$-poor magnetite $\left(<1 \mathrm{wt} . \% \mathrm{SiO}_{2}\right)$, ranging from epitaxial banding and overgrowths to more complex intergrowths [19]. Concentration of aqueous silica and Fe-silicate species in pore fluid enhance incorporation of Si during magnetite crystallization in BIF environments and the precipitates form via exsolution from the Si-bearing host magnetite at low temperature [29].

A variety of other elements $(\mathrm{Mg}, \mathrm{Mn}, \mathrm{Ca}, \mathrm{Zn}, \mathrm{Al}, \mathrm{Ti}, \mathrm{V}$, etc.) are associated with $\mathrm{Si}$ in hydrothermal magnetite, although subordinate in concentration. Their distribution can be shown as apparent zonation patterns when using microbeam techniques for imaging/mapping (EPMA or EDS-SEM), suggesting they can be associated with one another at the time of incorporation into magnetite (e.g. [34]). For example, a recent nanoscale study of "hydrothermal magnetite" from Los Colorados "magnetite-apatite" deposit, Chile [35] shows $\mu \mathrm{m}$-scale oscillatory zoning (seen on element maps for $\mathrm{Si}, \mathrm{Al}, \mathrm{Ca}, \mathrm{Mg}$, $\mathrm{Ti}$ and $\mathrm{Mn}$ ) that are tied to the presence of $\mu \mathrm{m}$ - to nanoscale inclusions of silicates and Ti-phases; no Si-magnetite nanoprecipitates were reported. Oscillatory zonation patterns relating to the occurrence of sub- $\mu \mathrm{m}$ scale inclusions have also been shown from magnetite-bearing ores on the margins of the Olympic Dam Iron Oxide Cooper Gold (IOCG) deposit, South Australia [36,37]. In a preliminary study of these magnetites [38], the presence of Si-magnetite nanoprecipitates with maghemite vacancy ordering was reported in the silician magnetite.

So far, however, no studies have reported silician magnetite as single grains from natural samples which were assessed down to the nanoscale. The only record of Si-magnetite with a spinel structure is from the synthetic studies at high $\mathrm{P}$ and $\mathrm{T}$ [16]. The disparity between the apparent homogeneity at the $\mu \mathrm{m}$-scale and marked nanoscale heterogeneity draws attention to the nature of the problem of defining what 'silician magnetite' actually is, and identifying the petrogenetic implications this would carry in any given geological environment. Constraining the nature of Si in magnetite is important for petrogenetic models as has been highlighted in previous studies. These include refining oxy-reduction conditions of ilmenite-magnetite thermometry at $\mathrm{T}>600{ }^{\circ} \mathrm{C}$ [14], understanding preservation of biosignatures in BIFs at $\mathrm{T}<300{ }^{\circ} \mathrm{C}$ [19], the stability of silician magnetite relative to pure magnetite, i.e., silician magnetite extends to more reducing conditions than pure magnetite, or the inhibition of oxidative transformation of silician magnetite into hematite [29]. Experimental and geochemical modelling studies have shown the possibility of $\mathrm{pH}$-controlled, non-redox transformation of magnetite into hematite (in some cases via transient maghemite) with major implications for supergene enrichment of BIFs [39,40]. Conceptual models for IOCG deposit genesis show regional and/or orefield zonation patterns resulting from fluids that evolve over time, or fluid-rock interaction ([41] and references therein). Generic changes in Fe-oxide speciation are stipulated, generally from magnetite to hematite, as the hydrothermal system evolves from alkali/calcic metasomatism (K-feldspar \pm biotite + calc-silicates) to hydrolytic (sericite/chlorite + quartz) alteration.

In this contribution, we undertake a comprehensive nanoscale study, using HAADF STEM imaging and STEM-EDS mapping and spot analysis, on magnetite from samples from the outer, weak mineralized shell at Olympic Dam. The study also included magnetite present as a magmatic accessory within the 
granite hosting the deposit. We aim to (i) characterize, and (ii) explore the petrogenetic value of silician magnetite from Olympic Dam for constraining ore genesis. The significance of nanoscale inclusions in hydrothermal and magmatic magnetite is also addressed. Considering the intrinsic presence of Fe-oxides within IOCG systems, this study has implications for IOCG-type mineralization and other types of hydrothermal deposits elsewhere. Lastly, the data presented here add new insights into the definition of silician magnetite and its occurrence in ore deposits.

\section{Geological Background and Sample Selection}

Olympic Dam is one of the largest $\mathrm{Cu}-\mathrm{Au}-\mathrm{U}-\mathrm{Ag}$ deposits worldwide [42] and is located in the Mesoproterozoic ( 1.6 Ga) IOCG province in the Eastern Gawler Craton, South Australia (Figure 1a). IOCG-type mineralization is affiliated with a major tectonomagmatic event at $\sim 1.6 \mathrm{Ga}$ that produced the Gawler Silicic Large Igneous Province (SLIP) [43]. Igneous activity is expressed as bi-modal volcanism and associated intrusive rocks: Gawler Range Volcanics (GRV) and Hiltaba Suite (HS), respectively. The large scale and high-metal endowment of IOCG provinces formed during the Precambrian Era are associated with assembly and break-up of the Columbia/Nuna supercontinent during which sublithospheric mantle fertilization of an older continental crust took place by extensive underplating of mafic magma [44].

The Olympic Dam deposit is located within a hematite-rich breccia complex ( $>5 \% \mathrm{Fe})$ in turn hosted by the Roxby Downs Granite (RDG) of HS affiliation (Figure 1b; [42] and references therein). High-precision, U-Pb ID-TIMS dating of magmatic RDG zircon (1593.87 $\pm 0.21 \mathrm{Ma}$ [45]) and of hydrothermal hematite $\left(1589.91 \pm 0.91 \mathrm{Ma}\right.$ [46]) show a difference of $\sim 3-4 \mathrm{Ma}\left({ }^{207} \mathrm{~Pb} /{ }^{206} \mathrm{~Pb}\right)$ between granite crystallization and IOCG mineralization. The RDG is a component pluton of the Burgoyne batholith; depth of emplacement is estimated at $6-8 \mathrm{~km}[47,48]$. The basement also comprises older rocks, including $\sim 1.85 \mathrm{Ga}$ Donington granitoids and $1.75 \mathrm{Ga}$ Wallaroo Group metasedimentary rocks [49]. Petrographic and nanoscale studies have identified alkali-calcic alteration in the RDG around the margins of the deposit [48,50-52]. Assemblages recognized include the presence of newly-formed, hydrothermal albite, Ba-rich K-feldspar, as well as calc-silicates (epidote and calcic garnet). Broader-scale alkali alteration is also documented from infrared reflectance spectroscopy mapping of drillcores across a $14 \mathrm{~km}$-long transect [53].

Olympic Dam displays mineralogical and geochemical zoning, both along the $\sim 6-\mathrm{km}$-strike and also throughout the $\sim 2 \mathrm{~km}$-depth in the SE part of the orebody [42]. Magnetite from deeper parts of the deposit is associated with an assemblage of pyrite + chalcopyrite, the outermost and deepest orezone. In contrast, hematite is present throughout all zones, including the inner- and uppermost bornite + chalcopyrite zone followed by the bornite + chalcopyrite zones. Hematite from the ore breccias, whether within clasts or the matrix, displays a variety of textures and geochemical signatures but the earliest hematite is oscillatory- and sectorial-zoned with respect to U, W, Sn and Mo [54]. This 'granitophile signature' is present throughout the strike and depth of the orebody and is clearly recognized from multivariate statistical analysis of a large lithogeochemical dataset [55]. Such hematite can also be reliable and accurately dated [46]. The geochemical signature and the age of the hematite are both evidence that mineralization is related to granite-derived fluids. The breccia hosting ore results from replacement of RDG and/or other lithologies that were unroofed during uplift and incorporated into the granite breccia, respectively. The observed overlap between the contours of the IOCG geochemical signature, as defined from principal component analysis [55], the $\geq 20$ wt. \% Fe isolines, and the sericite/K-feldspar boundary are all strong evidence that the RDG not only hosts mineralization but also likely provided the fluids for pervasive Fe-metasomatism and $\mathrm{Cu}-\mathrm{U}-\mathrm{Au}-\mathrm{Ag}$ mineralization. 
Table 2. Overview of samples.

Fample
Foil No. \#

Magnetite occurs throughout the weakly-mineralized (chalcopyrite + pyrite) granite that forms an outer shell is silician (EPMA analysis indicates the presence of $\mathrm{SiO}_{2}, \mathrm{CaO}$ and $\mathrm{MgO}$ at concentrations of up to $4.46,1.23$, and $0.91 \mathrm{wt}$. \%, respectively); relatively high concentrations of $U$ and REE were measured in silician magnetite from location \#1 and \#4 (Figure 1b) [37]. Formation of such magnetite in the outer shell is attributed to the earliest alkali-calcic alteration event [38] constrained at $\mathrm{T}>400{ }^{\circ} \mathrm{C}$ and as the $\mathrm{pH}$ changes from fields of $\mathrm{K}$-feldspar to sericite stability by geochemical modelling [37]. Recognition of silician magnetite (mottled patterns, high-Si) replacing magmatic magnetite (trellis textures, high-Ti, no-Si) led to the interpretation that measured concentrations of $\mathrm{Ca}$ and $\mathrm{Mg}$, aside from $\mathrm{Si}$, can be attributed to elements released from the granite during breakdown of magmatic feldspars and mafic minerals [37]. In contrast, magnetite from the massive interval on the northern side of the deposit was considered to be an inherited BIF-like unit based upon preliminary U-Pb LA-ICP-MS dating of coarse magnetite grains, which gave an age of $1.75 \mathrm{Ma}$ [56], and also the association with siderite [36]. 

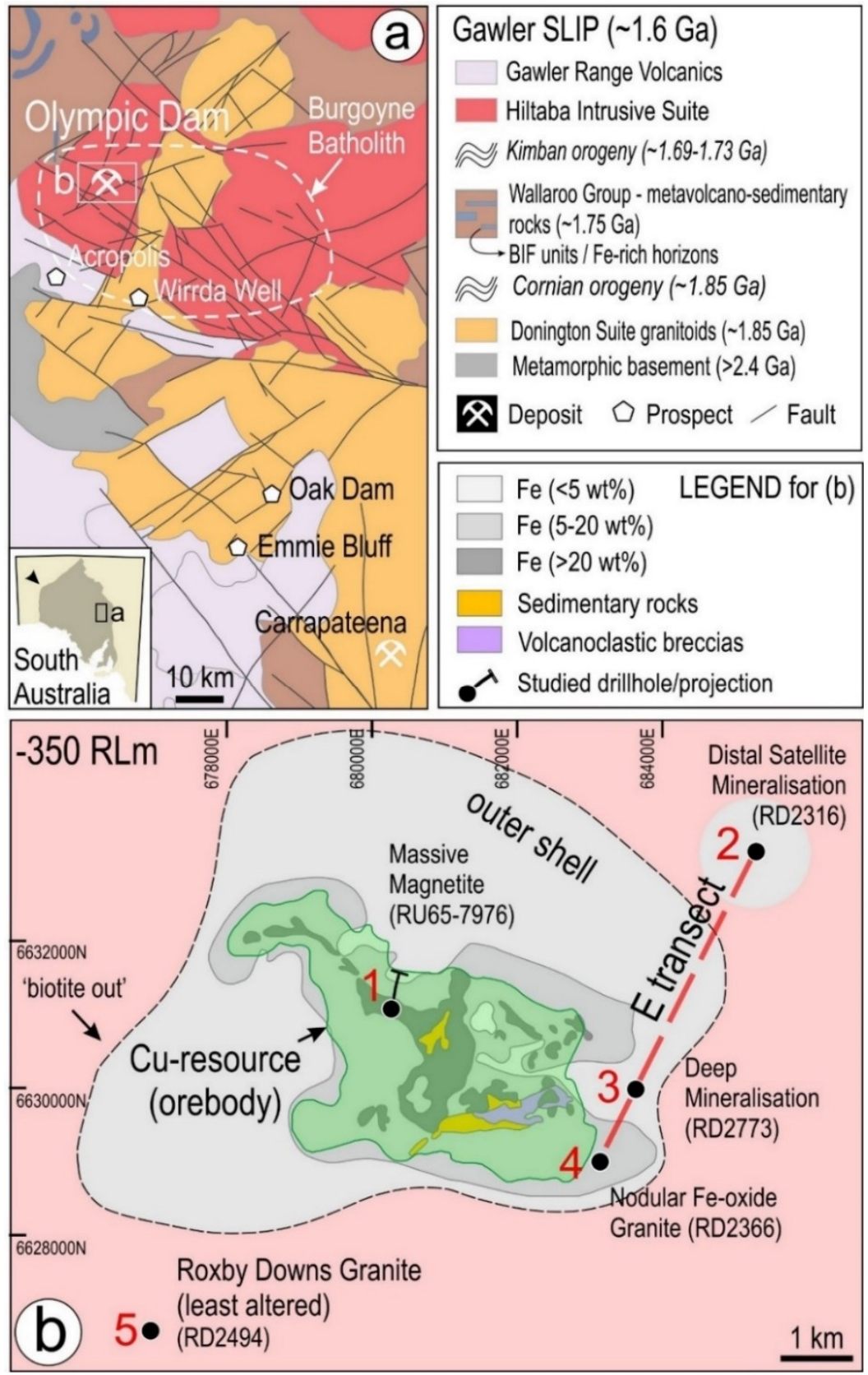

Figure 1. (a) Geological map of the Olympic Dam area outlining the main lithological units. Data sourced from https://map.sarig.sa.gov.au/ [49]. Inset shows location of this area in South Australia and extent of the Gawler Craton (arrowed). (b) Sketch of the Olympic Dam deposit showing main lithologies and projection of sampled drillholes (Table 2). SLIP-Silicic Large Igneous Province. Numbers 1-5 refer to sample locations (Table 2).

In the present contribution, we document silician magnetite from the same granite locations in the outer shell as those studied by Verdugo-Ihl et al. [37]. These samples are representative of a spectrum of Si-magnetite found in concentrations varying from massive intervals together with carbonate gangue to $\mathrm{cm}$ - to dm-sized nodules or irregular pockets in variably altered granite and at depths from $\sim 700 \mathrm{~m}$ to as much as $2 \mathrm{~km}$ (Table 2, Figure 1b). In addition, a sample of fresh RDG studied previously [48] is also included since it contains magmatic magnetite representative of that found at Olympic Dam. We consider these samples relevant for understanding the variation in mineralogical and geochemical characteristics of silician magnetite formed in granite-hosted IOCG systems. 


\section{Methodology}

Imaging was performed using a FEI Quanta 450 scanning electron microscope (SEM) (FEI, Hillsboro, OR, USA) with energy dispersive X-ray spectrometry and back-scatter electron (BSE) imaging capabilities. Element mapping was obtained using a Cameca SX-Five electron probe microanalyzer (Cameca SAS, Gennevilliers Cedex, France), running PeakSite Version 6.2 software for microscope operation, and Probe for EPMA software (distributed by Probe Software Inc. (Eugene, OR, USA). X-ray lines and standards for measurement of $\mathrm{Fe}, \mathrm{Si}$ and $\mathrm{Ca}$ were $\mathrm{Fe}-\mathrm{K} \alpha$ (almandine garnet), $\mathrm{Si}-\mathrm{K} \alpha$ (albite), and Ca-K $\alpha$ (apatite), respectively.

Foils for TEM study were prepared using a FEI-Helios nanoLab Dual Focused Ion Beam-SEM (FIB-SEM) (FEI, Hillsboro, OR, USA). Eight foils (Supplementary Materials Figure S1) were obtained from slices extracted in-situ and thinned (to $<100 \mathrm{~nm}$ ) by ion beam $\left(\mathrm{Ga}^{+}\right)$milling using a FEI Helios Nanolab 600 instrument (FEI, Hillsboro, OR, USA). The TEM foils were attached to Cu grids. EDS mapping and Bright Field (BF) STEM imaging was also performed on the FIB-SEM platform on some of the foils. Electron diffractions and imaging in BF-mode were performed on a Philips CM200 TEM operated at $200 \mathrm{kV}$ (Philips, Eindhoven, The Netherlands). The instrument is equipped with a LaB6 source, double-tilt holder and Gatan Orius digital camera (Gatan Inc., Pleasanton, CA, USA). Energy-dispersive X-ray spectra (EDS) were acquired using an Oxford Instruments X-Max 65T SDD detector running Aztec software (Oxford Instruments, Abingdon, UK). Image and diffraction measurements were performed using DigitalMicrograph ${ }^{\mathrm{TM}}$ 3.11.1 (Gatan Inc., Pleasanton, CA, USA).

HAADF STEM imaging and EDS spot analysis/mapping were performed using an ultra-high-resolution, probe corrected, FEI Titan Themis S/TEM operated at $200 \mathrm{kV}$ (FEI, Hillsboro, OR, USA). This instrument is equipped with the X-FEG Schottky source and Super-X EDS geometry. The Super-X EDS detector provides geometrically symmetric EDS detection with an effective solid angle of $0.8 \mathrm{Sr}$. Probe correction delivered sub-Ångstrom spatial resolution, and an inner collection angle greater than $50 \mathrm{mrad}$ was used for HAADF experiments using the Fischione HAADF detector. Diffraction indexing was conducted using Winwulff( 1.5 .2 software (JCrystalSoft, Livermore, CA, USA) and publicly available data from the American Mineralogist Crystal Structure Database. Crystal structure simulations were carried out using CrystalMaker®Version 10.1.1 (CrystalMaker Software, Begbroke, UK) and STEM for xHREMTM Version 4.1 software (HREM Research, Tokyo, Japan). All instruments used are housed at Adelaide Microscopy, The University of Adelaide.

\section{Results}

\subsection{Petrography}

Magnetite from the outer shell of the Olympic Dam deposit is characterized by the presence of $\mu \mathrm{m}$ - to $\mathrm{nm}$-scale inclusions of various composition and with variable distributions. At the $\mu \mathrm{m}$-scale, the distribution of these inclusions can define patterns of either intra-crystal oscillatory zoning, lamellar networks, or mottled areas of variable inclusion density (Figure 2). Crystal zoning is well-expressed in silician magnetite (Figure 2a) throughout all samples whereas magmatic magnetite from RDG shows lamellar networks of Ti-bearing oxides (ilmenite + rutile; Figure $2 b$ ) attributable to "trellis"-like exsolutions. An overlap of the two types of pattern within single grains, albeit fragmented, is observed in samples (Figure 2c) from the E transect. In this case, an overprint of an earlier magnetite of magmatic origin by Si-magnetite is recognized by the preservation of trellis domains superimposed by rhythmically banded patterns. The latter display morphological variation with inwards- and/or outwards-oriented rhythmic growth relative to the grain margins (Figure 2c). 


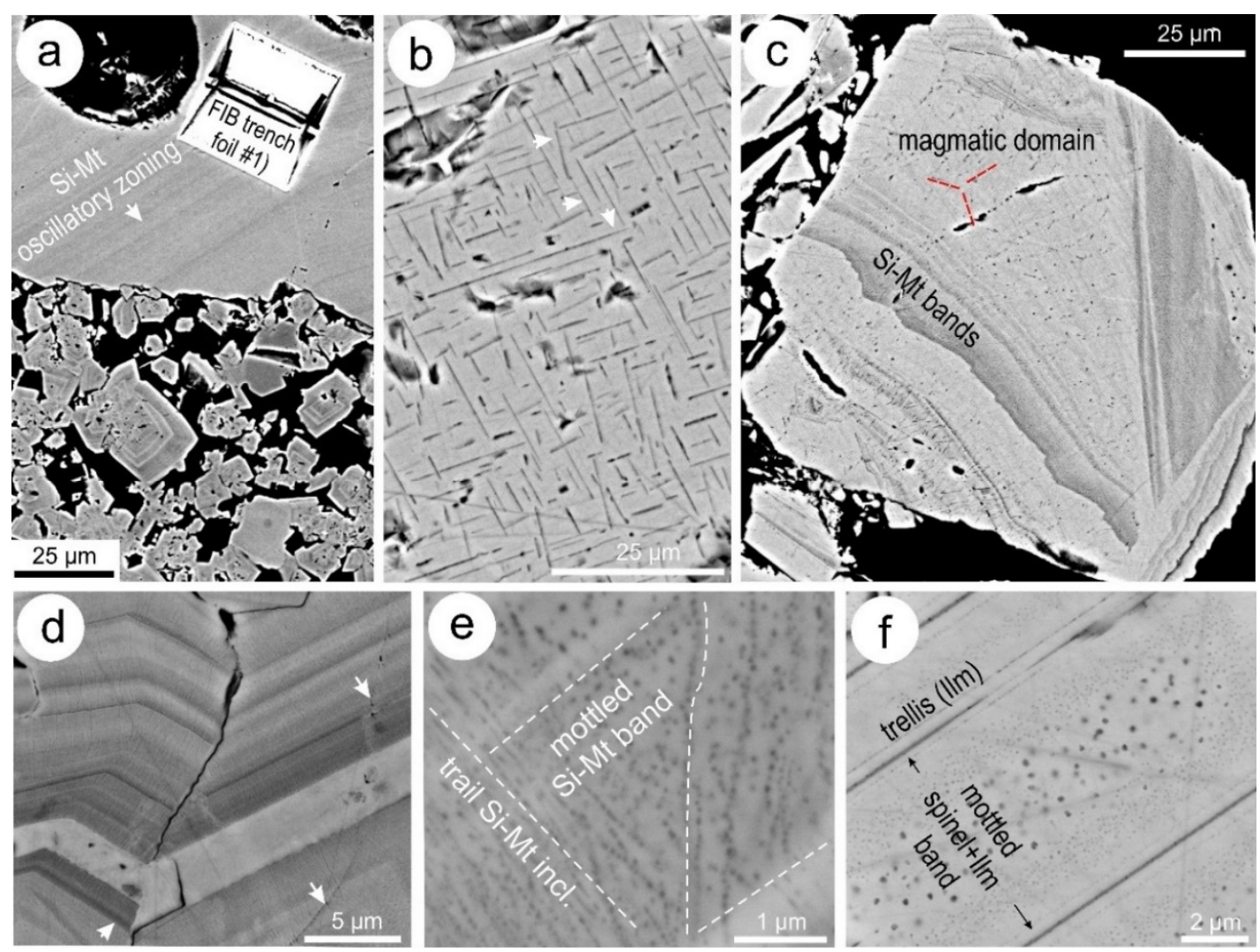

Figure 2. BSE images showing magnetite textures. (a) Coarse $(>500 \mu \mathrm{m})$ grain surrounded by small (a few $\mu \mathrm{m}$ to some tens of $\mu \mathrm{m}$ ) grains of silician magnetite (Si-Mt) typical for the massive magnetite interval (location \#1, Figure 1b). (b) Trellis exsolutions (white arrows) in magmatic magnetite from RDG (location \#5, Figure 1b). (c) Fragment of magnetite with magmatic domains superimposed by banded silician magnetite (location 5, Figure 1b). (d) Overprint of oscillatory zoning in silician magnetite observed as microfracturing and displacement of bands (white arrows). Mottled textures in silician and magmatic magnetite in (e) and (f), respectively. Note trails crosscutting crystal zoning in (e) and variation in inclusions size across any given band between sets of trellis exsolutions marked by ilmenite (Ilm).

Further morphological complexity is observed in the aggregates of silician magnetite whereby overprinting associated with micro-fracturing and disruption/reshaping of primary zonation patterns is recognizable within bands of highest contrast on the BSE images (Figure 2d). Such aspects are also seen as pervasive mottled textures crosscut by inclusion trails (Figure 2). The mottling is also present in the bands between the trellis textures in magmatic magnetite (Figure $2 b, f)$. This patterning defines internal domains of finer and coarser inclusions, suggesting that exsolutions are associated with thermal and/or chemical gradients relative to the trellis network (Figure 2f).

\subsection{Trace Element Distributions from Micron to Nanoscale}

EPMA mapping of Si-magnetite grains of medium size (100-300 $\mu \mathrm{m})$ from the massive magnetite interval in location \#1 (Figure 1b; drillhole RU 65-7976) shows oscillatory zoning with respect to variation in Si and Ca content (Figure 3). The latter can also vary in concentration from one grain to another (Figure 3a,b) but is always subordinate to $\mathrm{Si}$, as also shown by Verdugo-Ihl et al. [37] from EPMA spot analyses. EDS-SEM maps of one of the TEM foils (FIB-SEM platform) shows that the same elements are present throughout dense mottled areas (Figure 3c) but with a dot-like pattern rather than continuous appearance within a given band (Figure 3d). 

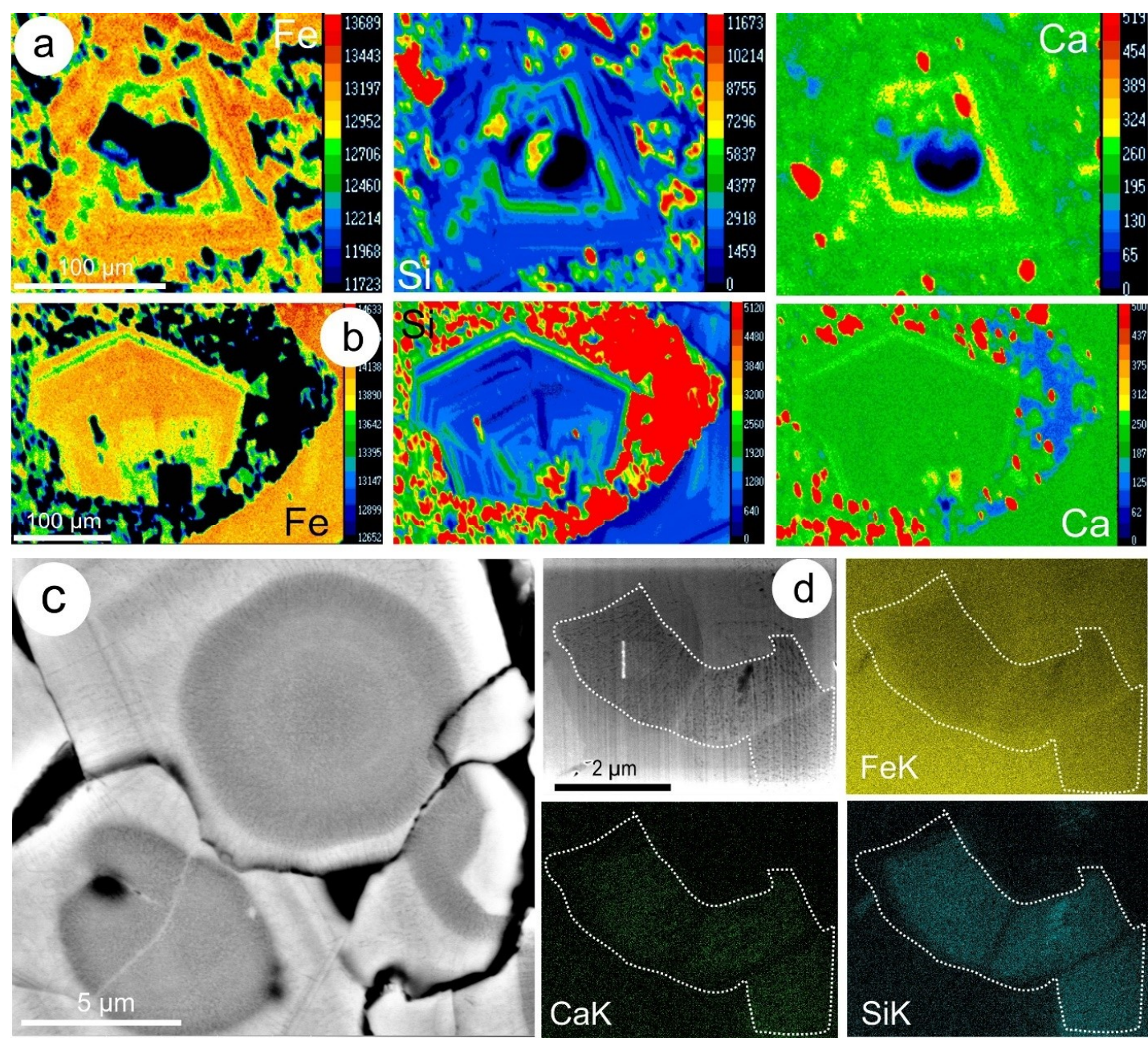
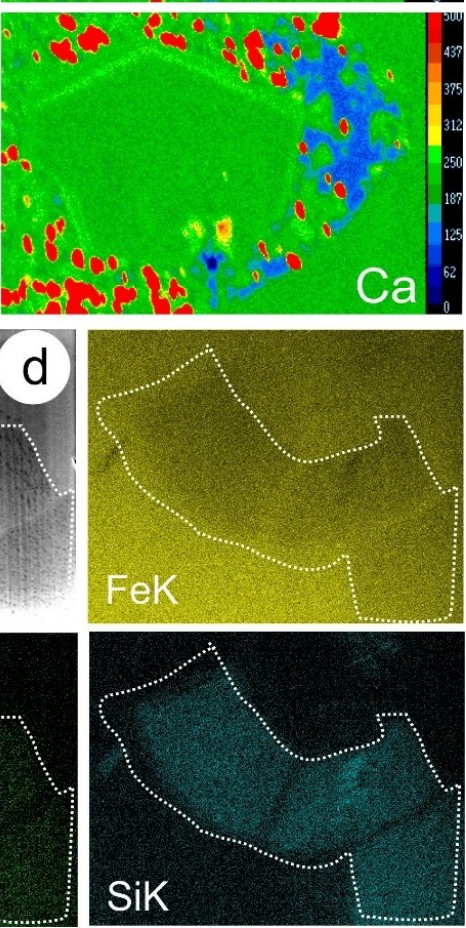

Figure 3. Element maps (EPMA in (a,b) and EDS-SEM (on the FIB platform) in (d)) and BSE image in (c) of medium-sized and small grains of silician magnetite (massive interval in location \#1; drillhole RU65-7976). Note the continuous appearance of the Si along the bands in (a,b). Note the fine-mottled texture in (c) becomes apparent on the maps in (d) which are obtained from a FIB-prepared foil. This illustrates the differences in maps depending upon the sample thickness, i.e., from the surface of a polished block and from $\mathrm{a}<100 \mathrm{~nm}$-thin foil in $(\mathbf{a}, \mathbf{b}, \mathbf{d})$, respectively.

The chemical patterns discussed above for silician magnetite reveal further chemical complexity when mapped at the nanoscale. This is expressed with respect to grains of different size in the same sample from the massive magnetite interval, or among specimens containing silician magnetite from different locations.

The inclusions present throughout the foil obtained from one of the less common, coarser grains ( $>500 \mu \mathrm{m}$ in size; foil \#1 obtained from the FIB trench in Figure 2a) within the massive magnetite interval are among the smallest (from a few to tens of nm; see also next section), not larger than nanoparticles (NP) (Figure 4a). They are only locally concentrated within the magnetite and no crosscutting inclusion trails are observed. In this case, each inclusion appears as an area of high-Si and low-Fe on EDS-STEM profiles and maps (Figure $4 \mathrm{~b}$ ). Other elements, notably $\mathrm{U}$ (in the smallest inclusions) and $\mathrm{Ca}+\mathrm{Mg}$ are also present throughout the mottled areas, albeit with very scarce distribution relative to the Si-bearing inclusions (Figure 4c). 

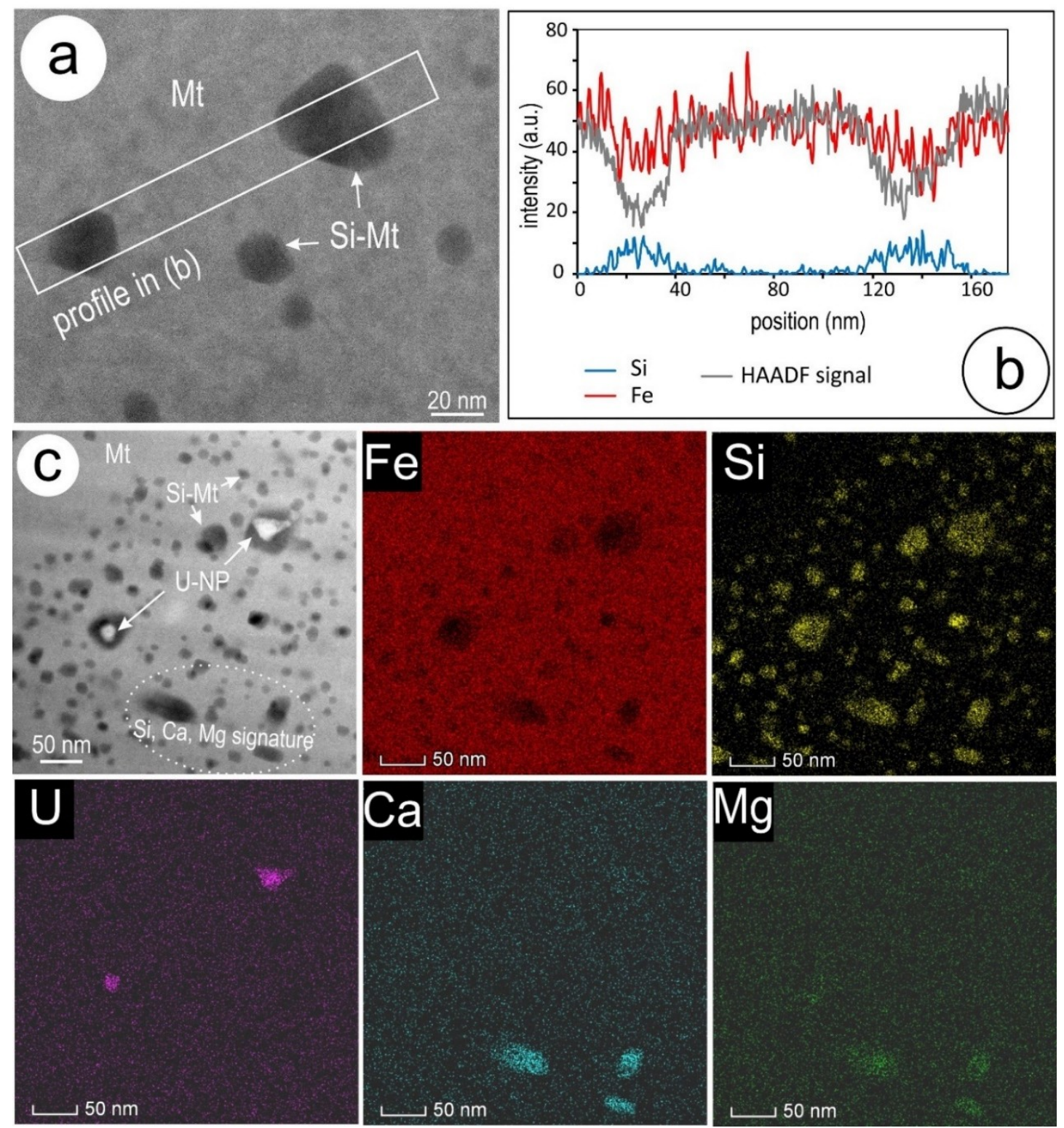

Figure 4. (a) HAADF STEM image and EDS-STEM profile (b) showing a field of Si-bearing nanoparticles (tens of nm; Si-Mt) in coarse magnetite (Mt) (foil \#1, FIB cut shown in Figure 2a). (c) Image and EDS-STEM element maps showing a field with denser inclusions in the same foil. Note that the dominant inclusions are Si-bearing (Si-Mt), whereas a minority are either U-bearing NPs or Si-Ca-Mg bearing (bright and dark on HAADF STEM image, respectively). The latter define a Si-Ca-Mg signature part of the nanoprecipitates attributable to silician magnetite.

Foils obtained from the medium-sized grains in the same sample $(150-200 \mu \mathrm{m})$ show the crosscutting relationships between crystal zoning and inclusion trails (Figure 5a,b). The crystal zoning features compositional variation with respect to the element association of individual bands, some of which are Si-rich whereas others display a prominent 3-element $(\mathrm{Si}>\mathrm{Ca}>\mathrm{Mg}$ ) association (Figure 5c). Low-magnification maps do not however highlight the smallest, bright inclusions occurring along the crosscutting trails that can be picked out on the HAADF STEM image (Figure 5b). 

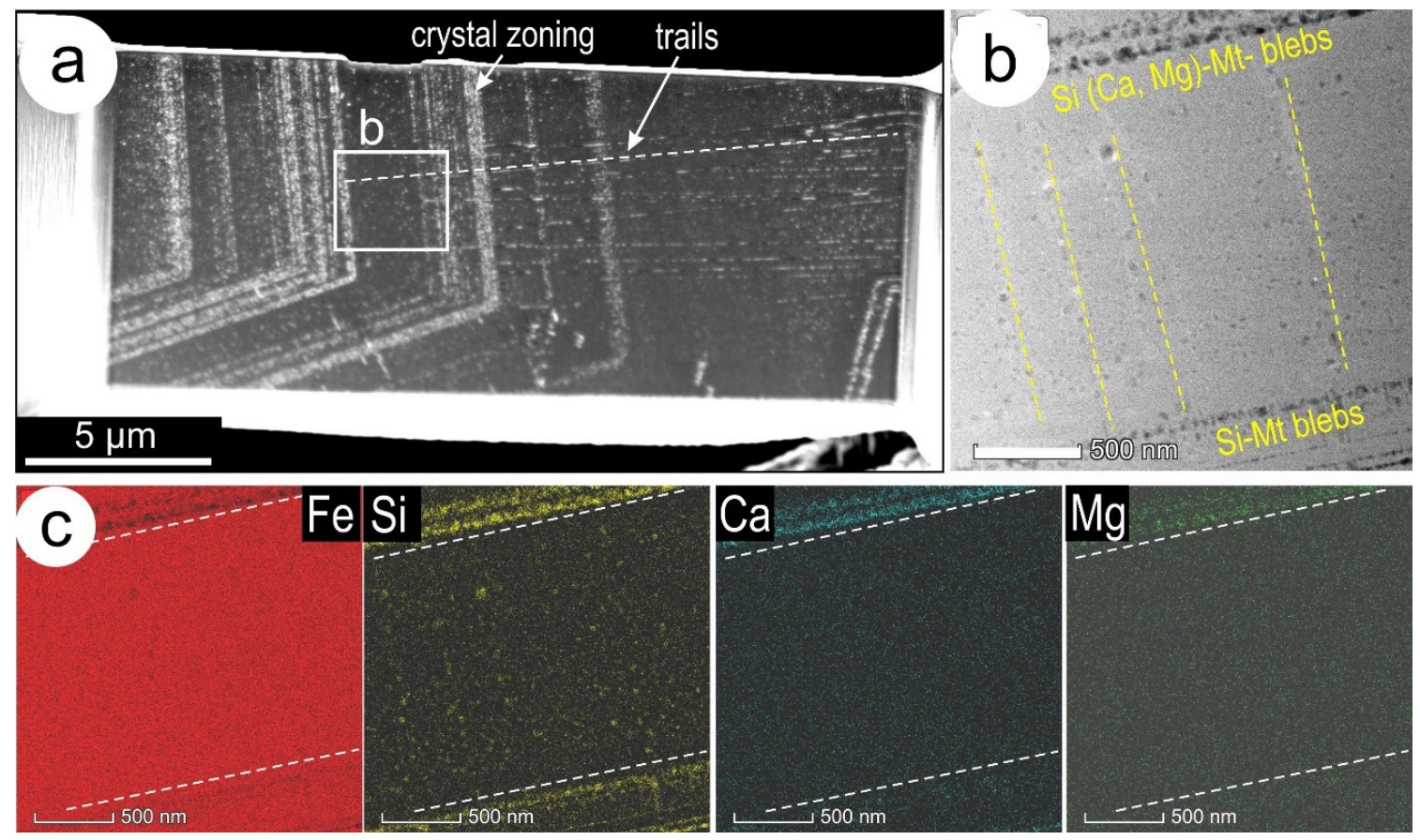

Figure 5. (a) Bright Field (BF) STEM image showing crystal zoning and crosscutting trails in medium-sized grains of magnetite (Mt) (foil \#2). (b,c) HAADF STEM and EDS maps showing variation in band composition for an area marked in (a). Note $\mathrm{Ca}$ and $\mathrm{Mg}$ are measured only in the top bands whereas the bottom bands are only Si, Fe-bearing corresponding to silician magnetite nanoprecipitates $(\mathrm{Si}-\mathrm{Mt}$ ) blebs. Note also that the bright inclusions present along the trails (dashed lines) are not depicted by the elemental maps at this resolution. The two bands with different chemistry are highlighted by white dashed lines on the maps.

Mapping at higher resolution confirms the Si-bearing dominant character of the inclusions in the bands defining crystal zoning (Figure $6 \mathrm{a}$ ), whereas the bright, $10 \mathrm{~nm}$-sized inclusions along the trails are identified as U-bearing nanoparticles (U-NPs) on element maps (Figure 6b). Such NPs are typically associated with $\mathrm{Si}$-rich areas in magnetite.

The correlation between $\mathrm{Si}, \mathrm{Ca}$ and $\mathrm{Mg}$ on the maps defines a 3-element association that can be attributed to the presence of calc-silicates. This becomes more pronounced in grains of Si-magnetite from samples representing the E-transect on the margin of the Olympic Dam deposit (Figure 7, Figure 8 and Supplementary Materials Figure S2). In addition, Ti and $\mathrm{Al}$ are also mapped at greater abundance in the same samples, indicating a more diverse association of calc-silicates and Ti-oxides (see below; Figure 7). The distribution of such inclusions varies from mottled (Figure 7a) to banded, with parallel orientation of acicular grains within a given magnetite band (Figure 7b, Supplementary Materials Figure S2). There is however, a sizable sub-population of inclusions which are only Si-bearing whereas others comprise $\mathrm{Si}+\mathrm{Al}$, or Ti-bearing inclusions as seen from the profile in Figure 7c. Superposition between $\mathrm{Si}, \mathrm{Ca}$ and $\mathrm{Mg}$ is nonetheless recorded across fields of calc-silicate inclusions (profile shown in Supplementary Materials Figure S2). 

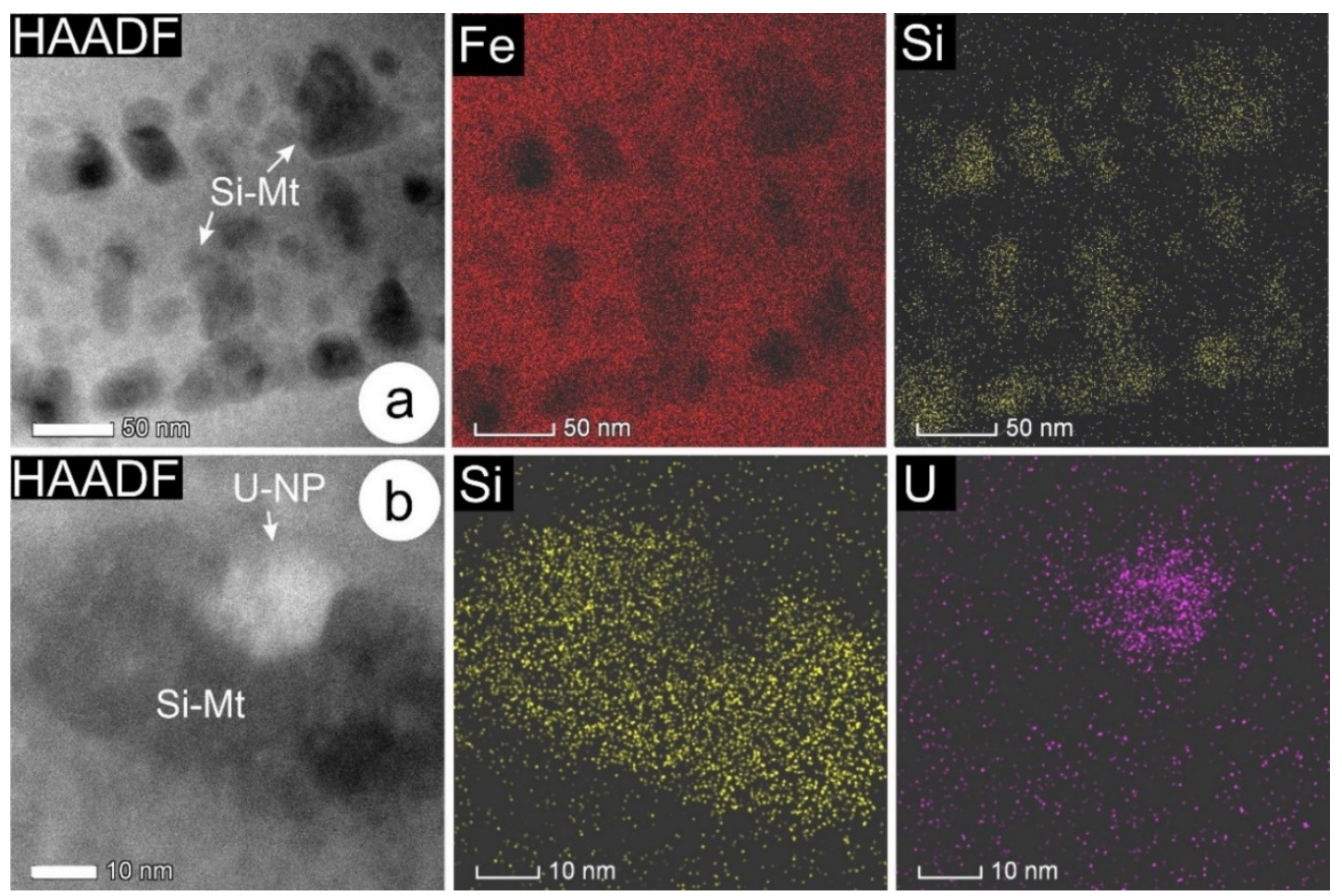

Figure 6. Higher-resolution HAADF STEM images (left) and EDS maps showing a field of Si-bearing nanoparticles (Si-Mt) along the crystal zoning in (a) and U-nanoparticles (U-NP) along trails crosscutting zoning in (b). Note U-NP is embedded within the Si-Mt nanoprecipitate.

The fine-scale crystal zoning becomes most complex in magnetite from the granite with nodular Fe-oxides (foil \#5; Figure 8). In this case, the bands comprise not only calc-silicates, quartz and $\mathrm{Si}-\mathrm{Fe}$-nanoprecipitates but also fine-scale zoning with respect to $\mathrm{K}$ and $\mathrm{Al}$. The density and size of the calc-silicates and Si-rich inclusions (quartz, Si-Mt nanoprecipitates) varies across the bands, albeit with a prominent Si-content in many inclusions (Figure $8 \mathrm{~b}$ ). In addition, the inclusions display a tendency towards two or three sets of orientation with angles between the orientations varying from $\sim 112^{\circ} / 68^{\circ}$ to $\sim 120^{\circ} / 60^{\circ}$ (Figure $8 \mathrm{a}$ ). The correlation between $\mathrm{Si}, \mathrm{Al}$ and $\mathrm{K}$ is shown by high-resolution mapping of some of the thin, elongated, wide rods in another magnetite sample from the same location (foil \#7; Supplementary Materials Figure S3). This type of inclusion may represent sericitic mica formed from a precursor potassium feldspar.

The two types of patterns attributable to overprinting of magmatic magnetite by silician magnetite are distinct in terms of type of inclusions, i.e., Al-bearing spinel (hercynite) + ilmenite and calc-silicates + rutile, respectively (Figure 9a,b). As shown above for all other examples of magnetite, there is a sub-population of Si-Fe-nanoprecipitate inclusions, generally the smallest, no more than a few $\mathrm{nm}$ in width. Some of these can be Al- and K-bearing (Supplementary Materials Figure S3). The boundary between the two domains is also marked by a relative increase in $\mathrm{Si}$ as shown on the profile in Figure 9c. The population of Ti-bearing inclusions increases in such cases relative to silician magnetite elsewhere. The association between different types of inclusions in each domain is exemplified by profiles across such assemblages (Figure 9d,e). In the silician magnetite domain there is a clear separation between the three types of inclusions, rutile, calc-silicates, and Si-Fe-nanoprecipitates. In the magmatic domain of the grain, assemblages of hercynite + ilmenite or ilmenite + rutile represent the main association. 

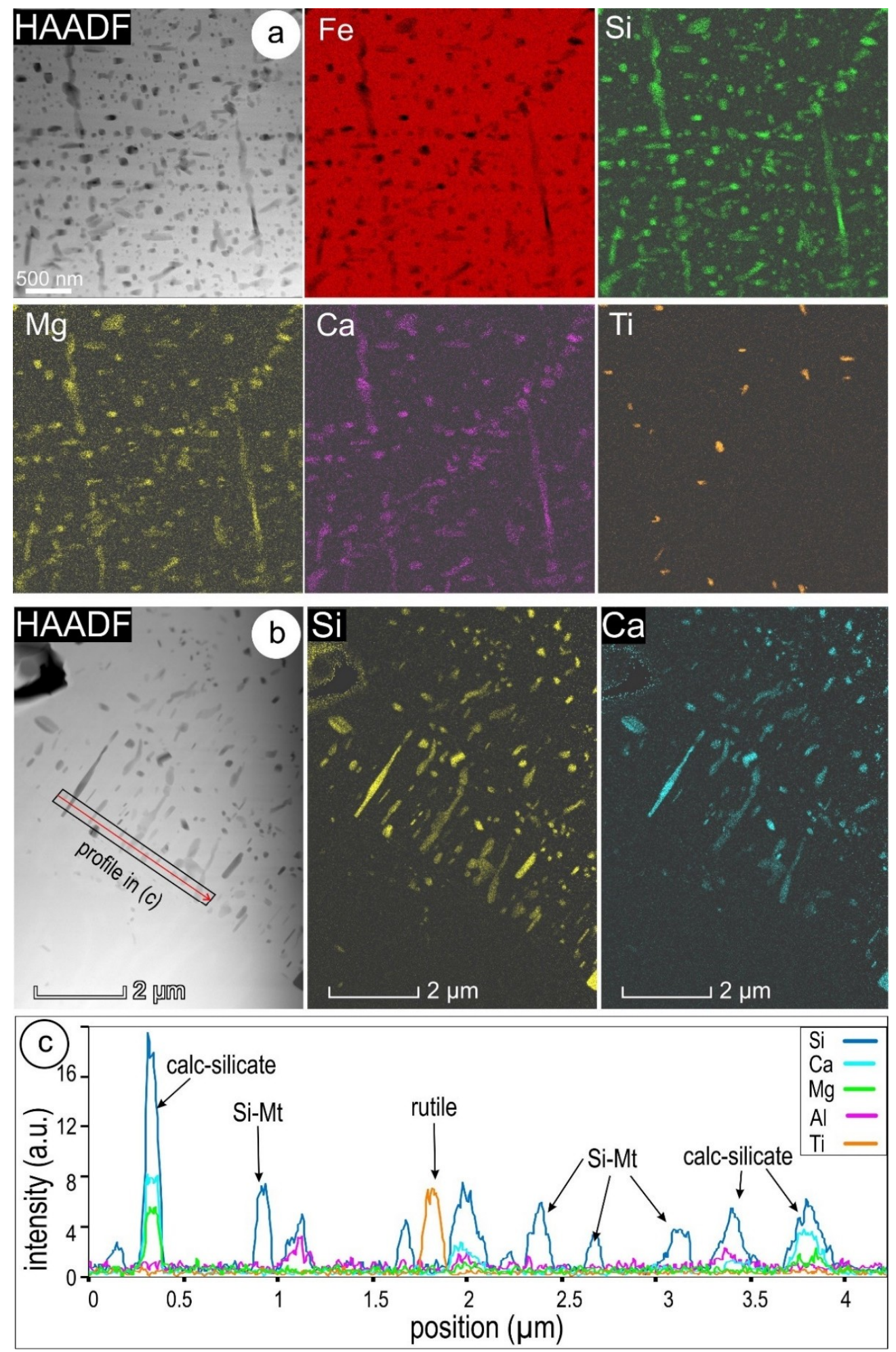

Figure 7. HAADF STEM images (left side) and STEM EDS maps showing mottled area with fine particles in magnetite (Distal Satellite Mineralization, location \#2; foil \#3) in (a), and acicular + bleb inclusions in magnetite (Deep Mineralization, location \#3; foil \#4) in (b). (c) Profile across fine particles in (b) showing the varied character of the inclusions. Overlap between $\mathrm{Si}, \mathrm{Ca}$ and $\mathrm{Mg}$, typical of the acicular inclusions, is indicative of calc-silicates, whereas the bleb-like particles are either Si- or Ti-bearing particles (rutile). The Si-bearing particles are attributable to Si-Fe-nanoprecipitates (Si-Mt) with low $\mathrm{Si} / \mathrm{Fe}$ ratio. 

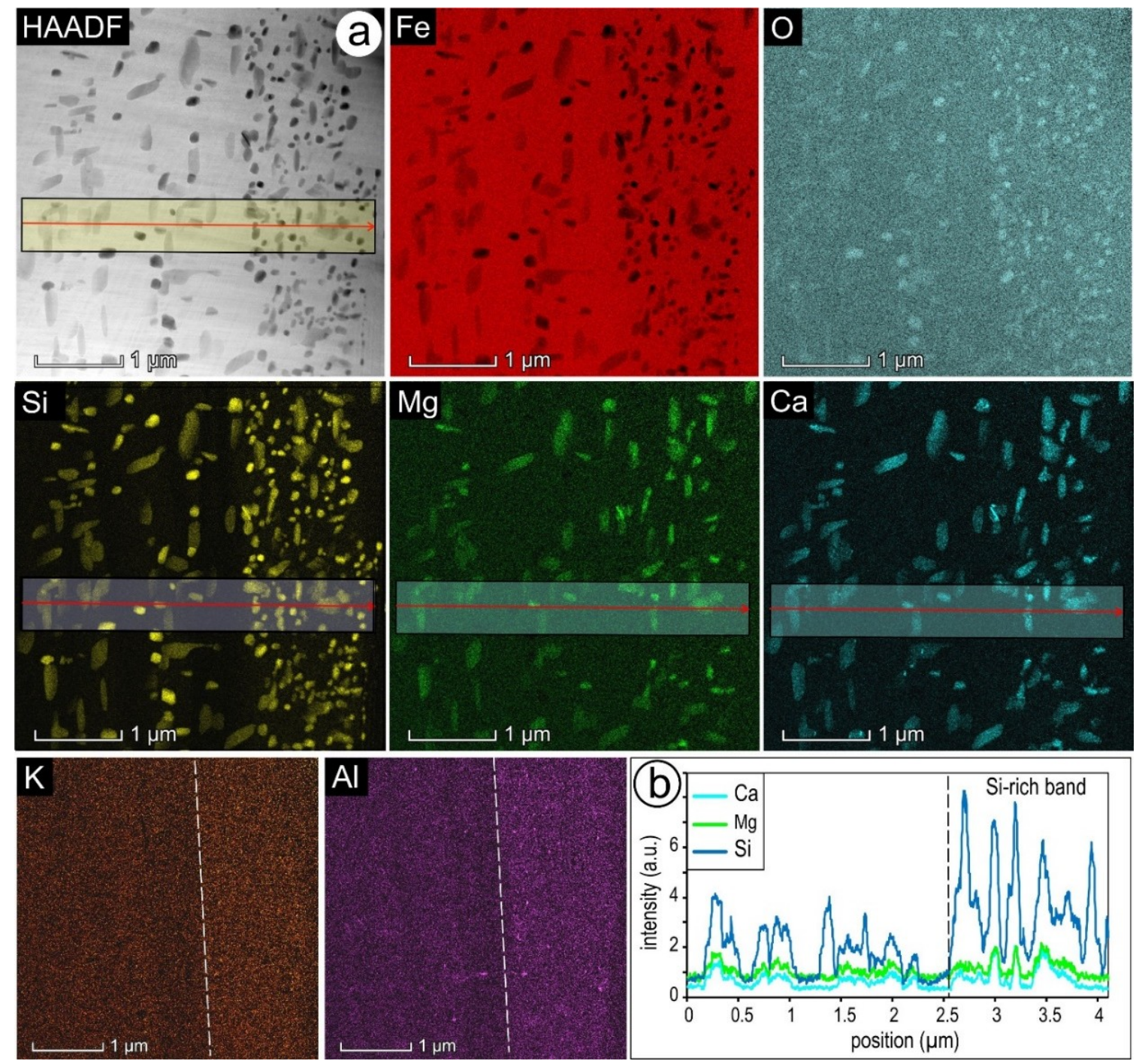

Figure 8. (a) HAADF STEM (left side) image and STEM-EDS maps showing the presence of inclusions within magnetite (location \#4, E transect; foil \#5). Note variation in size and shape of inclusions with element concentration, whereby the smallest, bleb-like are highest in Si and Mg-, Ca-bearing ones are fine- to coarser acicular inclusions (calc-silicates). In contrast $\mathrm{Al}$ and $\mathrm{K}$ show banding, with slightly higher concentration overlapping with the band with densest inclusions. (b) Profile across particles in (a) showing sharp variation in Si signal across the contact between the bands with variable size/density inclusions. There is however, no to little variation in the $\mathrm{Ca}$ and $\mathrm{Mg}$ signals corelating with the fact that many of the Si-high blebs are $\mathrm{SiO}_{2}$ (quartz?). 

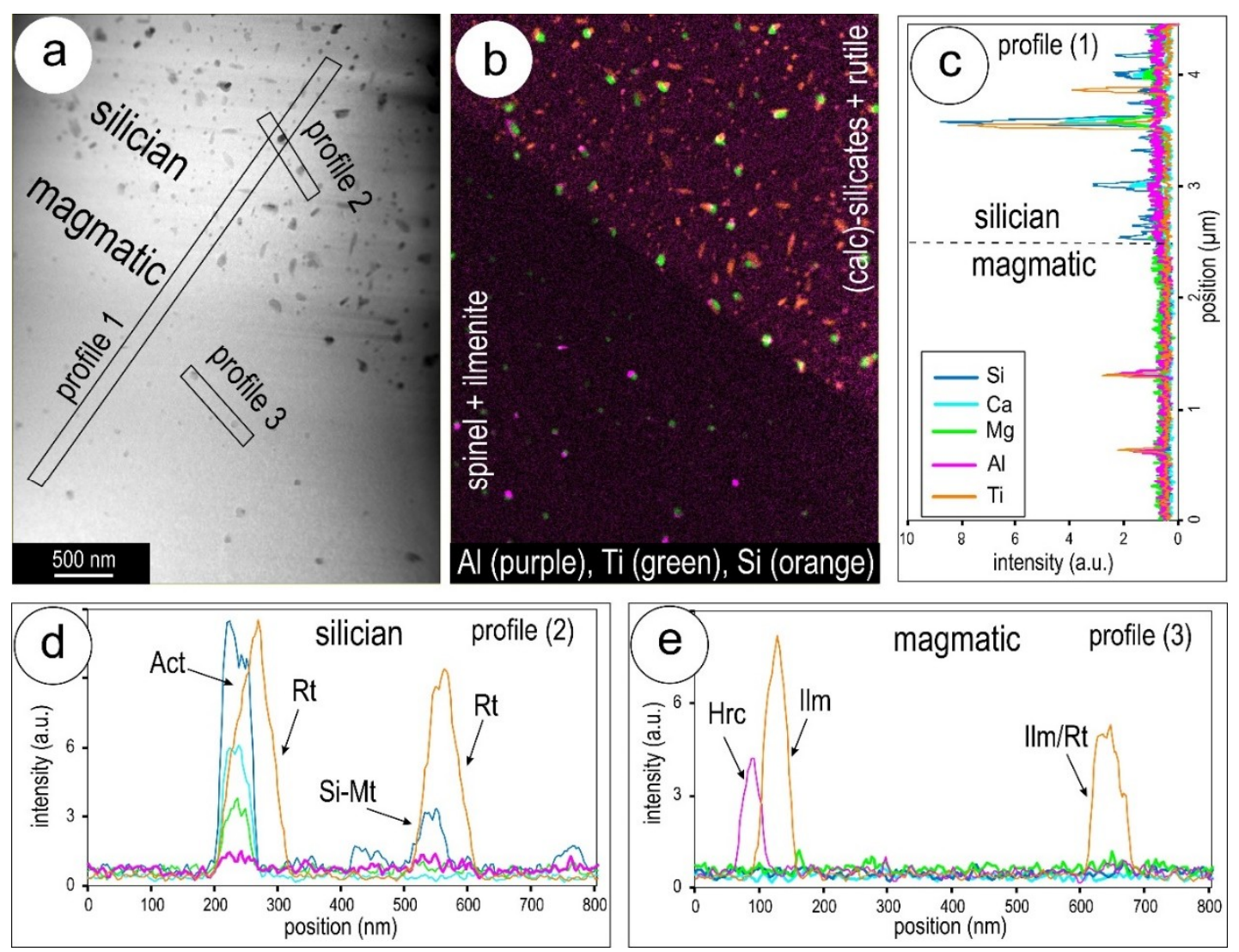

Figure 9. (a) HAADF STEM image of magnetite with silician and magmatic domains as marked (foil \#6). Location of profiles 1-3 is also shown. (b) Overlap between Al, Ti and Si STEM-EDS maps showing the presence of distinct inclusions in the two domains as marked. (c) Profile across the two magnetite domains showing the sharp increase in $\mathrm{Si}, \mathrm{Ca}, \mathrm{Mg}$ signals in the silician domain, whereas $\mathrm{Al}$ is higher in the magmatic domain. The Ti signal is high in both but can be highest in the silician domain due to coarser rutile inclusions. (d,e) Profiles across inclusions from the silician and magmatic domains. Note presence of actinolite (Act), Si-Fe-nanoprecipitates (Si-Mt) and rutile (Rt) in (d). In (e), the Al-bearing spinel hercynite ( $\mathrm{Hrc})$ and ilmenite (Ilm) form composite inclusions.

In general, inclusions from the magmatic domain are smaller than those in the silician magnetite domain. Nonetheless, coarser inclusions, tens of $\mathrm{nm}$ in size, also occur along trails of limited length (Supplementary Materials Figure S1). In this case, 3-component assemblages comprising rutile/ilmenite (?), hercynite $\left(\mathrm{FeAl}_{2} \mathrm{O}_{4}\right)$ and a $\mathrm{Zn}-\mathrm{Al}-\mathrm{Fe}-$ bearing-phase were found (Figure 10). It is interesting to note the conspicuous $\mathrm{Zn}$-signature in the magmatic domain, which contains a spinel with composition between franklinite $\left(\mathrm{ZnFe}_{2} \mathrm{O}_{4}\right)$ and gahnite $\left(\mathrm{ZnAl}_{2} \mathrm{O}_{4}\right)$ as inferred from the spectrum (Figure 10). The spectrum obtained for the Ti-rich species indicates the presence of $\mathrm{Fe}$, inferring that rutile may have formed from pre-existing ilmenite. 


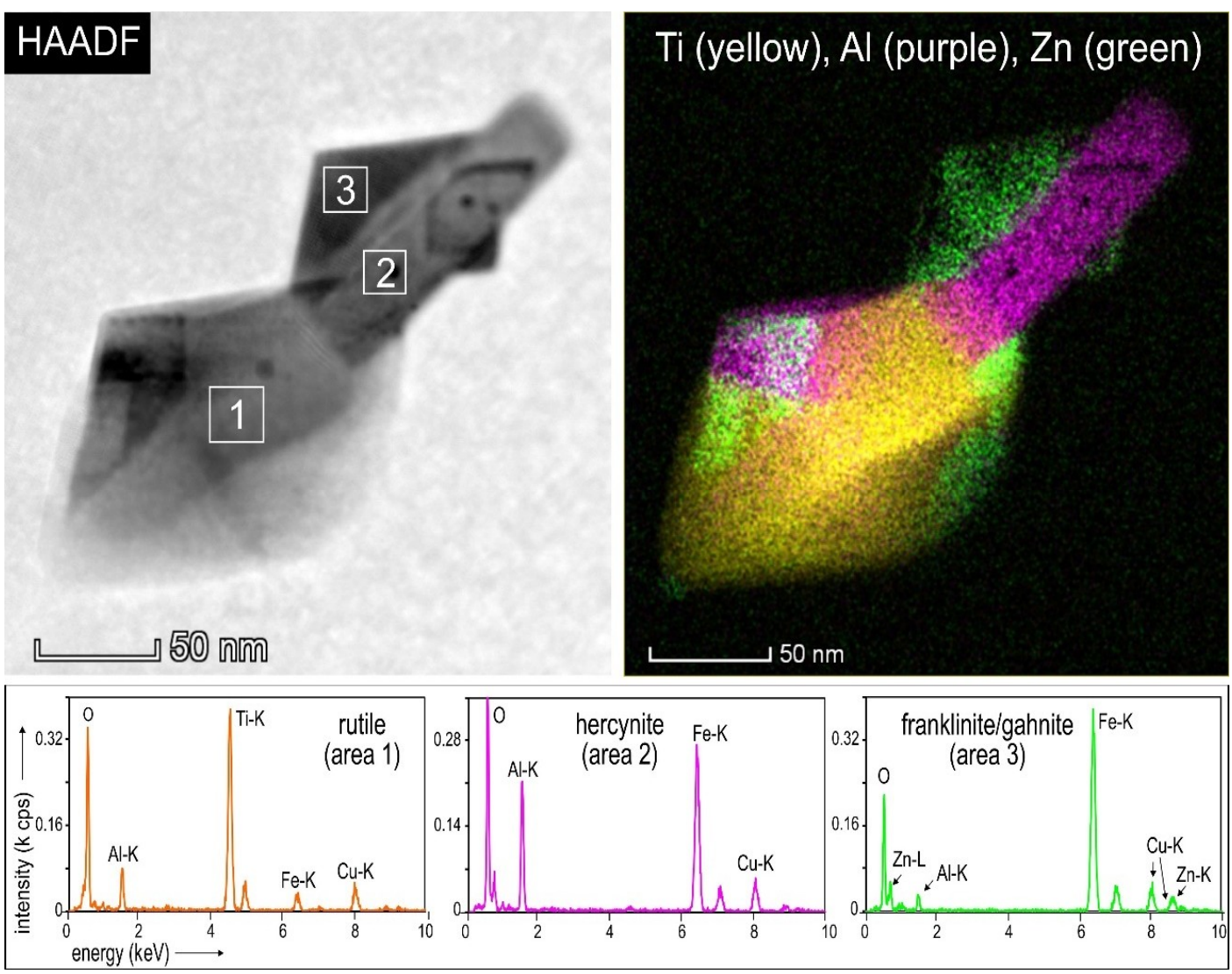

Figure 10. HAADF STEM (left side) image and overlap between Ti, Al and Zn STEM-EDS maps showing a multi-component inclusion in the magmatic domain of magnetite from foil \#6. Spectra for the inclusions collected from areas numbered 1-3 on the HAADF STEM image are shown underneath. The Cu signal is from the TEM grid.

Mottled textures in magmatic magnetite from host granite at Olympic Dam (foil \#8; Supplementary Materials Figure S1) comprise two populations of inclusions: coarser (tens of $\mathrm{nm}$ ); and smaller (no more than a few $\mathrm{nm}$ in size) with random distribution relative to one another (Figure 11a). The overlap between the distributions of Al, Ti and Fe shows a binary association between Ti- and Al- species within the coarser inclusions, with a dominance of the Ti-phases (Figure 11b). Spectra obtained from such inclusions indicate that some of these also include ulvöspinel, in addition to ilmenite and hercynite (Figure 11c). The spectrum representing host magnetite shows no other elements present. 

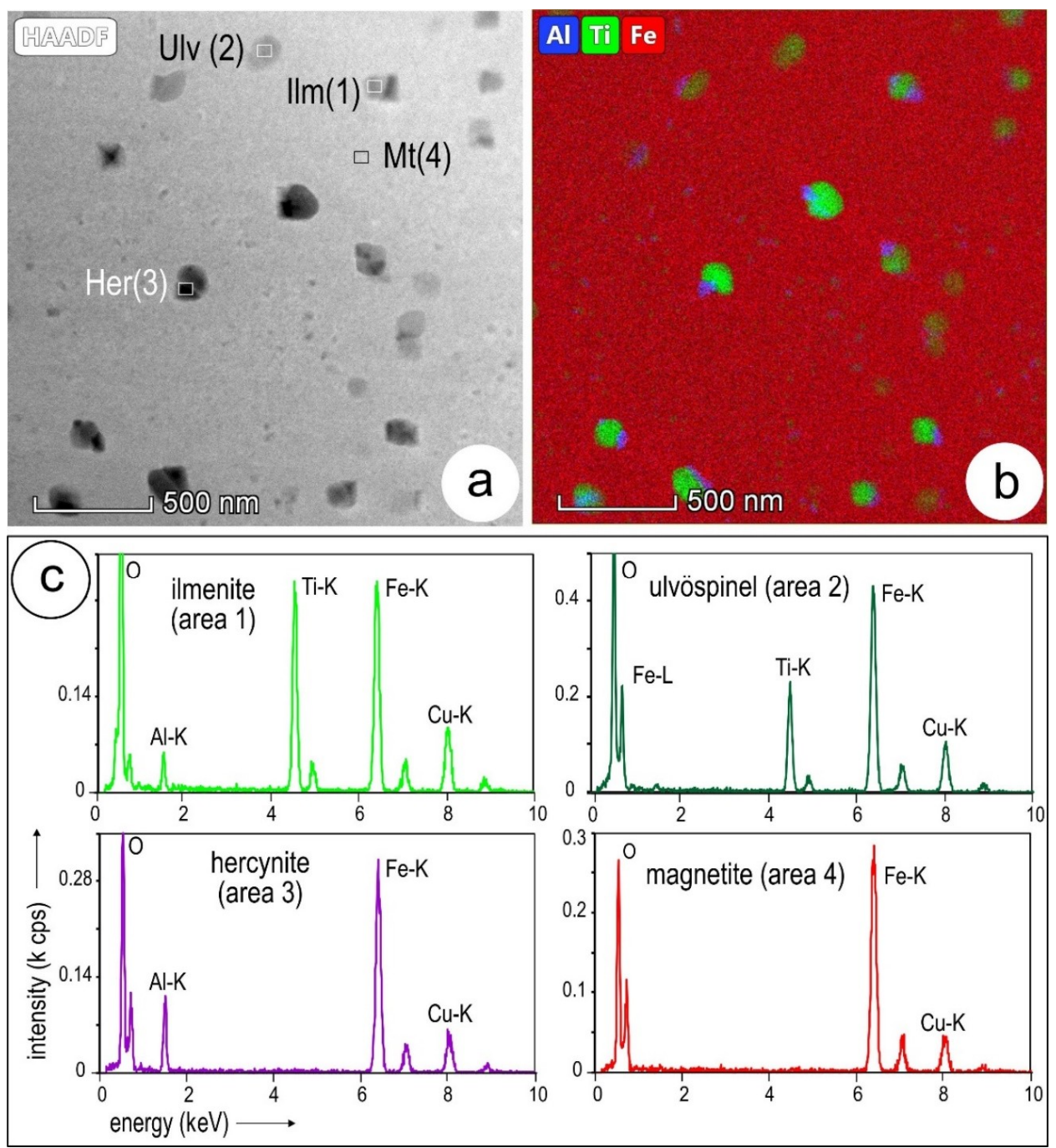

Figure 11. HAADF STEM image (a), STEM-EDS maps (b) and spectra for bleb-like inclusions and host magnetite (Mt) (c) typical of magmatic magnetite from least-altered RDG (location \#5; foil \#8). (a) Mottled texture with inclusions of two sizes, fine particles and nanoparticles. (b) Overlap between $\mathrm{Al}$, Ti and Fe maps showing the coarser blebs are Ti- and Al-bearing (binary inclusions). (c) Spectra of inclusions and host magnetite obtained from areas marked in (a). Note the presence of ulvöspinel (Ulv) in some of the blebs (lower HAADF signal intensity in (a)). This shows the inclusions comprise two compositionally distinct spinels, Ti- and Al-bearing, respectively, associated with ilmenite (Ilm). Note that the host magnetite does not contain measurable Ti or Al. The Cu signal is from the TEM grid. Her-hercynite.

\subsection{Ilmenite and Spinel Inclusions in Magmatic Magnetite}

HAADF STEM imaging of bleb-shaped inclusions in magmatic magnetite provides further insights into the phase assemblages of hercynite, ulvöspinel and ilmenite (Figure 12a,b). In detail, boundaries between all three spinel species (hercynite, ulvöspinel and host magnetite), show epitaxial relationships, i.e., parallel orientations of main crystal axes, indicative of growth under equilibrium during exsolution (Figure 12c). High-resolution imaging of magnetite and ilmenite on [100] zone axis (Figure 12d,e) is obtained with the specimen at the same tilt, thus implying they are oriented coherent to one another. The atom distribution on the HAADF STEM images is assessed by STEM simulations and crystal-structure models after identifying the zone axis using Selected Area of Electron Diffractions (SAED) or Fast Fourier Transform (FFT) (Figure 12f,g). The bright dots on magnetite ${ }_{[100]}$ represent Fe in either octahedral $(\mathrm{M})$ or tetrahedral $(\mathrm{T})$ positions, whereby the $\mathrm{T}$ site appears slightly fainter 
that the M site (Figure 12f). The dumbbell-shaped bright dots on ilmenite ${ }_{[100]}$ correspond to Fe atoms, whereas the lighter $\mathrm{Ti}$ atoms shown on the crystal model and simulation are not resolved by the present imaging (Figure 12g).
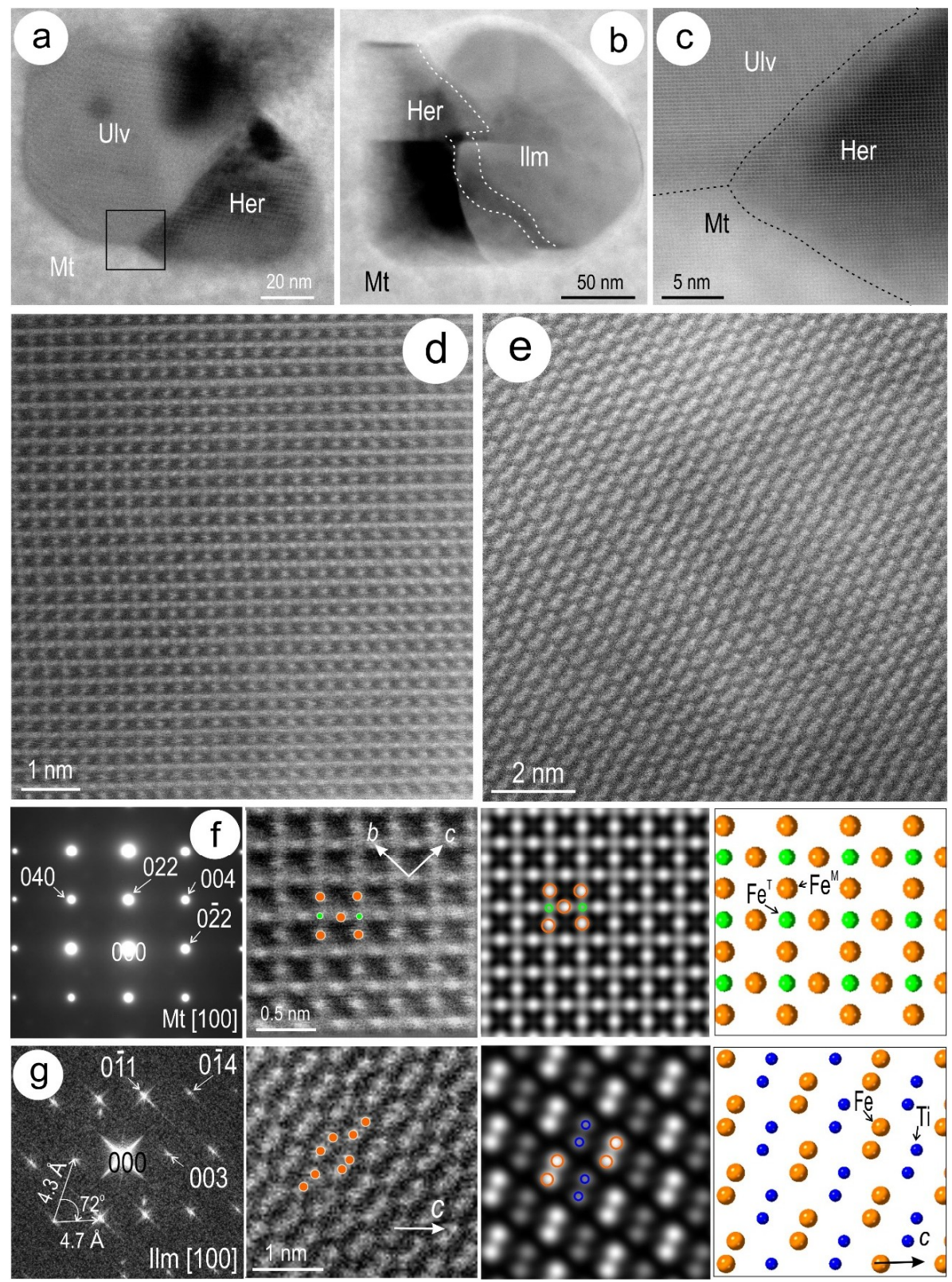

Figure 12. HAADF STEM images (a-e) showing associations between inclusions in magmatic magnetite (foil \#8). These show binary associations between the Ti- and Al-spinels, ulvöspinel (Ulv) and hercynite (Her) in (a) or hercynite and ilmenite (Ilm) in (b). The darkest areas in (a,b) are holes produced during the FIB milling. Epitaxial relationships between the phases and host magnetite (Mt) is shown in (c). (d,e) High-resolution images showing atomic arrangements in magnetite and ilmenite on [100] zone axis in each phase. (f,g) From left to right: indexed selected area of electron diffraction (SAED), HAADF STEM image, STEM simulation and crystal model for magnetite and ilmenite on [100] zone axis. In magnetite, $\mathrm{Fe}$ atoms in $\mathrm{M}$ and $\mathrm{T}$ sites are imaged as spots of different size and intensity. In ilmenite, only the Fe atoms are imaged as dumbbells arrays. The lighter Ti atoms are not captured in the present images. 


\subsection{Calc-Silicates and other Mineral Inclusions in Silician Magnetite}

An eclectic range of nanomineral inclusions with sizes ranging from fine particles (FP) to NP were identified in the silician magnetite using EDX-STEM spot analysis/mapping, and high-resolution HAADF STEM imaging. These assemblages comprise silicates, carbonates, rutile, and U-, W- and Y + As-bearing NPs (Figures 13 and 14; Supplementary Materials Figures S4 and S6). Calc-silicate ( \pm rutile) inclusions are present in magnetite from the eastern side of OD (E transect, locations 2-4) whereas U-, W-, Y-As-NPs were only identified from massive magnetite on the northern side in location \#1 (Figure 1b).
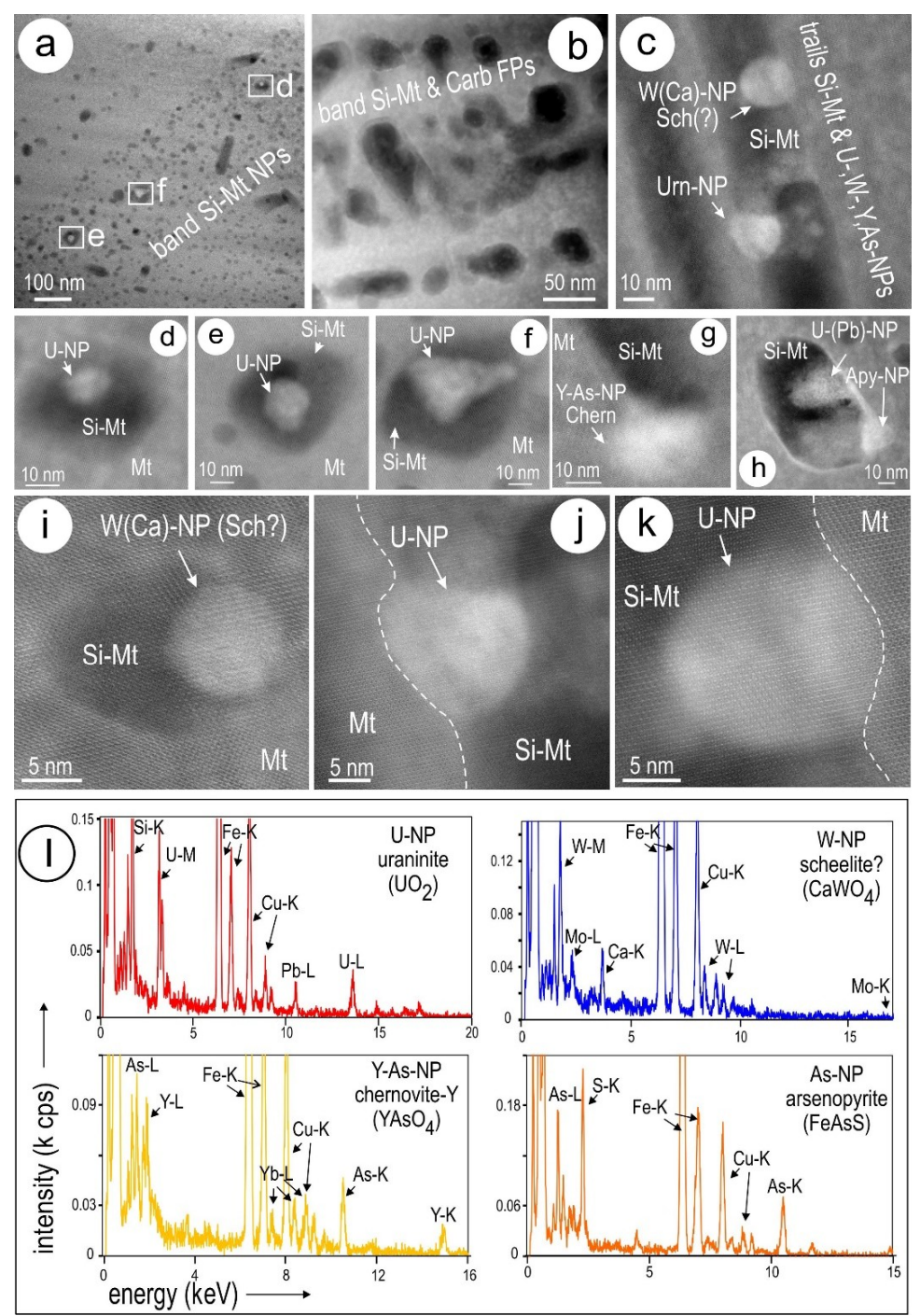

Figure 13. HAADF STEM images (a-k) and STEM-EDS spectra (1) showing U-, W-, Y-As-, As-NPs associated with Si-Fe-nanoprecipitates \pm carbonates (foils \#1 and \#2). Note the finest NPs, mostly U-bearing (uraninite), occur along the crystal zoning in the coarser magnetite (a; $d-f$; foil \#1) whereas the more diverse NPs occur along cross-cutting trails in the medium sized grains of magnetite (c; g-k; foil \#2). In all cases the NPs are embedded within Si-Fe-nanoprecipitates (Si-Mt). Apy-arsenopyrite; Chern-chernovite; Sch-scheelite; Urn-uraninite. The Cu signal is from the TEM grid. 


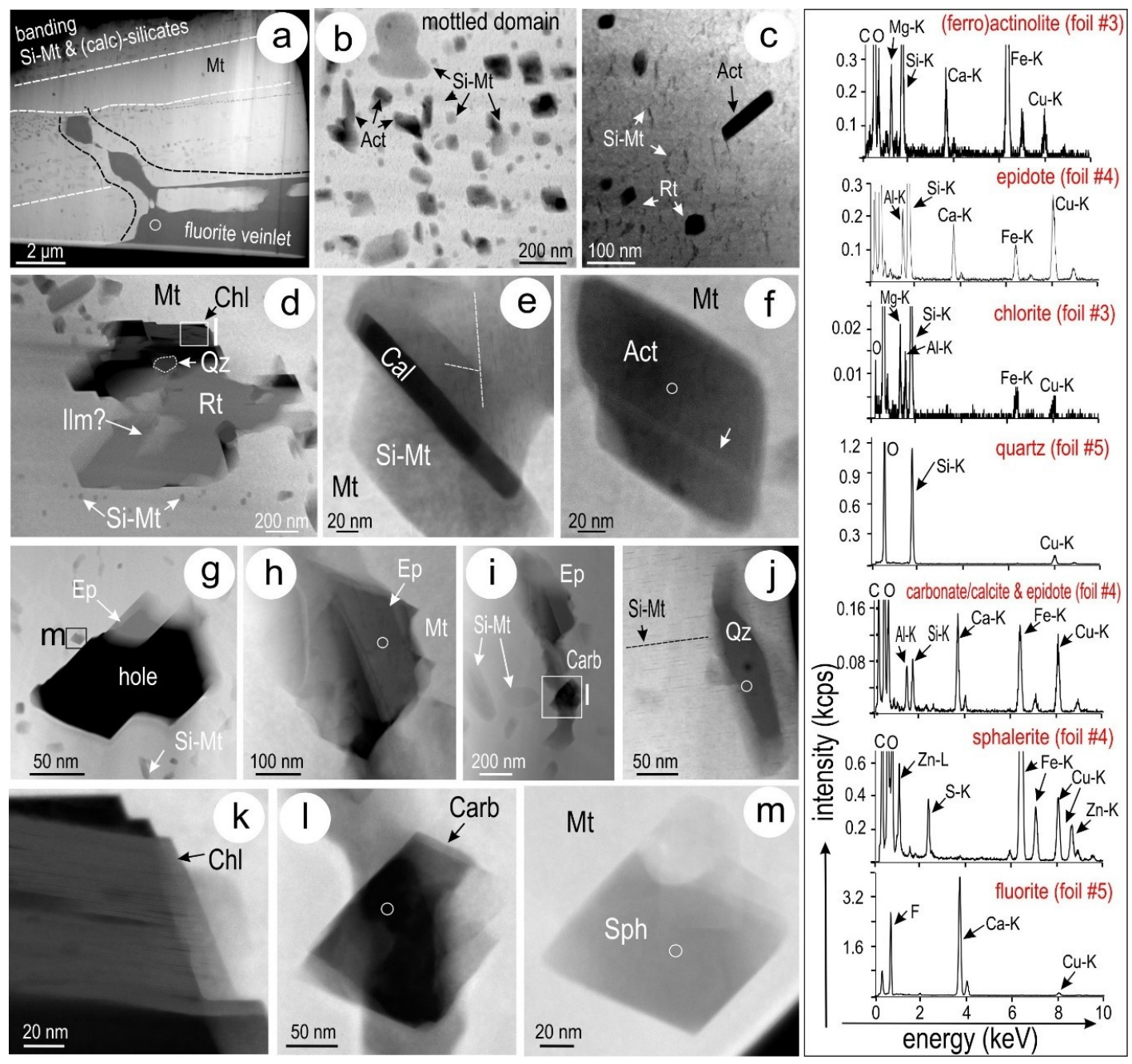

Figure 14. HAADF STEM images (a-m) and STEM-EDS spectra (to the right; as marked) showing mineral inclusions in silician magnetite from the eastern transect (foils \#3-5). Textures in silician magnetite displaying banding and overprint by vein fluorite in (a), mottled blebs with actinolite (Act) and Si-Fe-nanoprecipitates (Si-Mt) in (b), and difference in sizes and orientation between Act and Si-Mt in (c). (d) complex assemblage in one of the coarsest inclusion clots (foil \#3) showing an eclectic assemblage with rutile (Rt), ilmenite (?, Ilm), chlorite (Chl), quartz (Qz) indicative of inherited associations from a precursor magnetite of magmatic origin (?). (e) Coarser Si-Mt bleb with a rod of calcite (Cal). (f) One of the largest Act inclusions with internal zoning most likely due to Fe/Mg variation. Note the inclusions is not associated with $\mathrm{Si}-\mathrm{Fe}$-nanoprecipitates. (g-i) Composite associations of epidote and carbonates along arrays of Si-Mt. The hole in ( $\mathbf{g}$ ) is produced by FIB milling. (j) One of the coarsest $\mathrm{SiO}_{2}$ inclusions superimposed by strips of $\mathrm{Si}-\mathrm{Mt}$ (dashed line). It is not clear if these are amorphous or crystalline $(\mathrm{Qz})$, could not obtain reliable SAEDs from these inclusions. (k-m) Close-up images showing details of some of the rarer inclusions. Note the layered structure of Chl in (k), the rhombus-like shape of carbonate (Carb) and sphalerite in (l) and (m), respectively. The $\mathrm{Cu}$ signal on the spectra is from the TEM grids.

In the massive magnetite, the distribution, speciation and size of inclusions vary in the same sample (polished block) depending on the grain size of the host magnetite (Figure $13 \mathrm{a}-\mathrm{c}$ ). The NPs are very rare in the coarsest grains (Figure 13a) but are relatively abundant along trails crosscutting crystal zoning in the medium-sized grains (Figure 13c). The few U-NPs found among the Si-Fe-nanoprecipitates defining the crystal zoning in the coarsest grains occur as single blebs with spherical or lobate shapes and are typically embedded within the Si-Fe-nanoprecipitates (Figure $13 \mathrm{~d}-\mathrm{f}$ ). In contrast, carbonate inclusions are found among the clusters of Si-Fe-nanoprecipitates defining the zoning in medium-sized magnetite 
(Figure 13b), whereas the U-, W- and As-Y-bearing NPs are present along trails crosscutting crystal zoning (Figure 13c,g-k). In the latter case, the NPs are attached to the rod- and bleb-shaped Si-Fenanoprecipitates forming the trails. Arsenic-NPs were identified either as chernovite or arsenopyrite (Figure 13g,h); the latter clusters together with U-NPs (Figure 13h). Single, spherical blebs with sizes varying from $\sim 10 \mathrm{~nm}$ to several tens of $\mathrm{nm}$ are typical of $\mathrm{W}$ - and U-NPs (Figure 13i-k). It is worth noting $\mathrm{Ca}$ and Mo in the W-NPs suggesting these are scheelite. Although the element maps for both types of magnetite grains show the presence of $\mathrm{Ca}$ and $\mathrm{Mg}$ in some of the Si-rich inclusions (Figures $4 \mathrm{c}$ and $5 \mathrm{~b}$ ) the spectra obtained from such areas show that these elements are very minor relative to Si and Fe.

A diverse range of mineral inclusions were identified throughout the banding and from mottled domains in magnetite from all other locations along the E transect (Figure 14). In such cases, replacement of magnetite by secondary minerals (e.g., vein fluorite; Figure 14a) is also observed. Calc-silicates, represented by (ferro)actinolite, in some cases also associated with precursor calcic clinopyroxene (diopside-hedenbergite series) and epidote (Deep Mineralization) are prevalent. In contrast to the northern location \#1, these calc-silicates are generally coarser and can form acicular inclusions throughout the mottled areas or occur crosscutting and oblique to fine rods of Si-Fe-nanoprecipitates nanoprecipitates (Figure $14 \mathrm{~b}, \mathrm{c}$ ). Rutile is the second most abundant mineral and can occur as stubby grains interspersed with calc-silicates (Figure 14c) or tied to micron-sized clots with a diverse mineralogy including zircon, ilmenite (?) and secondary chlorite (Distal Satellite; Figure 14d). Calcite rods are found in association with Si-Fe-nanoprecipitates (Figure 14e). Coarser, euhedral grains of actinolite show compositional variation on HAADF STEM images (Figure 14f). Epidote is present on the margins of some of the largest voids, or forms part of elongate multi-component clusters including other minerals (Figure 14h,i). Quartz is particularly abundant throughout the magnetite with two sets of Si-Fe-nanoprecipitates (foil \#5; Figure 14j). Typical morphologies for the identified inclusions include lamellar stacks of chlorite, euhedral, rhombohedral carbonates and rare sphalerite (Figure 14k-m). Representative spectra for these minerals are shown in Figures 13 and 14.

Identification of calc-silicate and Ti-mineral inclusions present throughout the Si-magnetite was assessed by HR-HAADF STEM imaging combined with STEM simulation and crystal structure models on zone axes calculated from FFTs (Figures 15 and 16, and Supplementary Materials Figures S4 and S5). Imaging of one of the coarser inclusions identified as (ferro)actinolite from EDS STEM mapping and spot analysis (Supplementary Materials Figures S4 and S5) shows a greater complexity. This is expressed as lattice-scale intergrowths between calcic amphibole and clinopyroxene (diopside-hedenbergite series) on [011] zone axis in each mineral (Figure 15a,b) and with the specimen tilted on the $[11 \overline{2}]$ zone axis in host magnetite. The transition from pyroxene to amphibole is observed along the (1111) directions in the two minerals with a scalloped boundary marked by atom disorder (Figure 15a). Stacking intergrowths between the two minerals are also observed along the $a$ axis (Figure 15b). The epitaxial relationship on [011] zone axis is facilitated by the comparable angles and distances on lattice vectors in each of the two minerals as marked on Figure 15c. Differences in cation distributions along $(11 \overline{1})$ directions, i.e., denser for the amphibole relative to the pyroxene, are concordant with crystal models for the two minerals (Figure 15d-f) and provides an easy way to discriminate the two minerals. The bright banding in the (ferro)actinolite grain in Figure $14 \mathrm{f}$ might be associated with a comparable lattice-scale stacking between the calcic clinopyroxene and amphibole, as shown in the example above. 

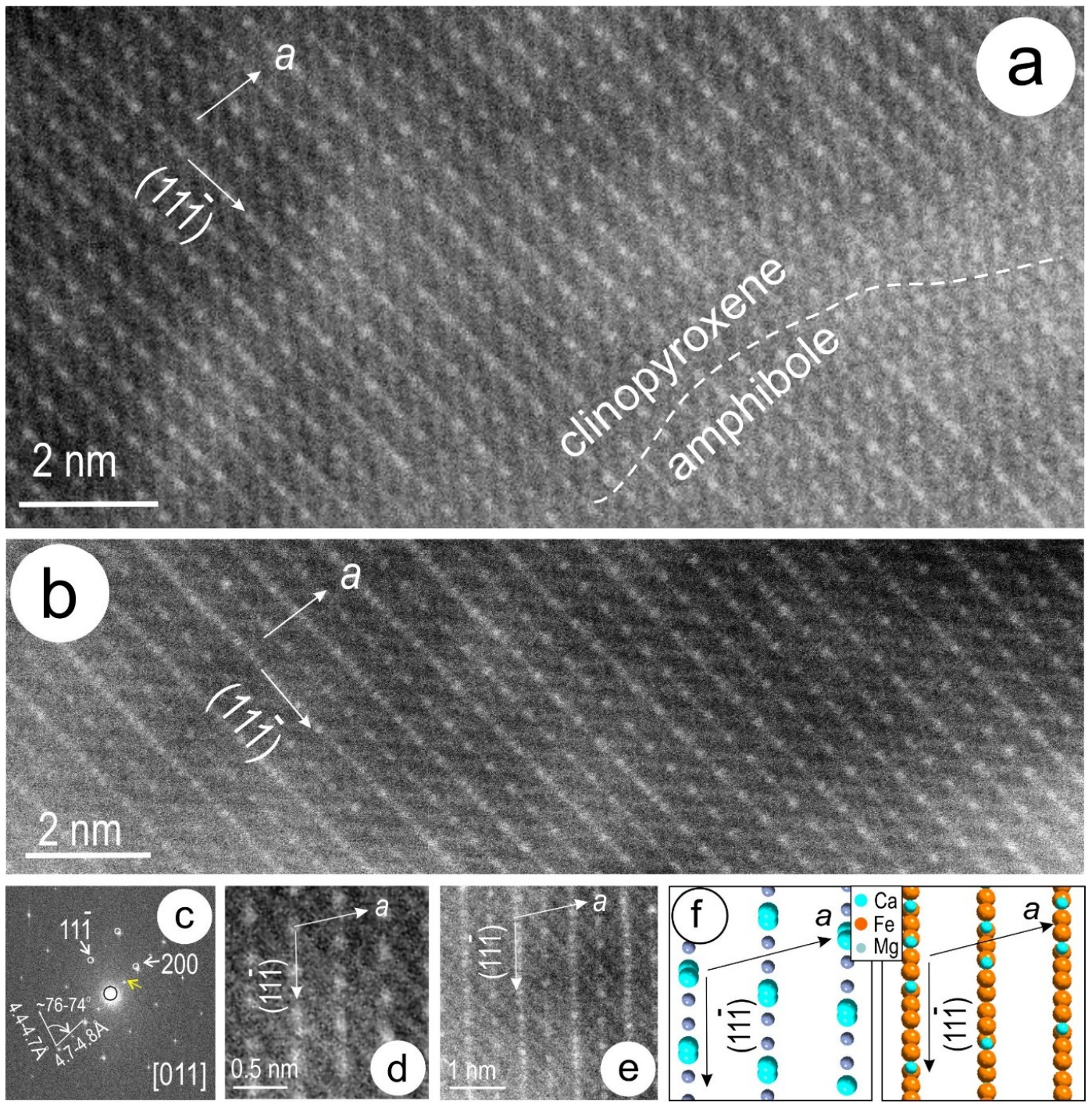

Figure 15. (a,b) High-resolution HAADF STEM images showing stacking intergrowth between calcic clinopyroxene and amphibole on [011] zone axis (inclusion from Supplementary Material Figure S4, foil \#4). (c) Fast Fourier Transform (FFT) of image in (a) showing diopside and actinolite on [011] zone axis. Note the satellite reflections along $a^{*}$ (yellow arrow) corresponding to the amphibole. The two phases have comparable angles at $76^{\circ}$ and $74^{\circ}$, respectively, between the lattice vectors $(11 \overline{1})^{*}$ and $a^{*}$. (d-f) Images and crystal structures corresponding to diopside and actinolite on [011] zone axis. Bright atoms correspond to cations with denser packing on $(11 \overline{1})$ for the amphibole (e and right on $\mathbf{f}$ ) relative to the clinopyroxene ( $\mathbf{d}$ and left on $\mathbf{f}$ ).

Atomic-scale images of two epidote (Ep) inclusions, both hosted by the same magnetite grain, were obtained on two zone axes at different specimen tilts $\left(\mathrm{Ep}_{[011]}\right.$ and $\mathrm{Ep}_{[\overline{101]}}$ at tilt on $\mathrm{Mt}_{[11 \overline{2}]}$ and $\mathrm{Mt}_{[1 \overline{1} 0]}$, respectively; Figure 16). Irregular, dark bands on HAADF images (Figure 16a) correlate with the presence of satellite reflections and streaks along $a^{*}$ axis on the FFT for [011] zone axis in epidote (Figure 16c). There is a good fit between the high-resolution image and the crystal structure of epidote on this zone axis. This is relevant for discriminating the sorosilicate structure of epidote, which comprises a ring of double $\mathrm{Si}_{2} \mathrm{O}_{7}$ and single $\mathrm{SiO}_{4}$ tetrahedra (Figure 16e,f). The bright spots on the image can be attributed to overlap between metals (Fe, Ca, and $\mathrm{Al}$ ) and Si-tetrahedra (Figure 16f, bottom). The image also shows that the orientation of the tetrahedra rings are flipped/twinned at $180^{\circ}$ to one another with irregular steps along the $a$ axis (motifs highlighted in yellow and red on Figure 16e). This stacking disorder is also expressed as streaks along $a^{*}$ on the FFT image (Figure 16c). The dark 
bands on the lower magnification image (Figure 16a) can therefore be attributed to observed nm-scale twinning combined with variation in the Al:Fe ratio. There is also a good fit between the HAADF image and the STEM model (Figure 16g,h). The [212] zone axis in epidote displays much tighter atom packing on $(\overline{101})$ direction (Figure $16 \mathrm{~b}, \mathrm{~d}$ ), which is also reproduced by the STEM simulation (inset, Figure 16i).
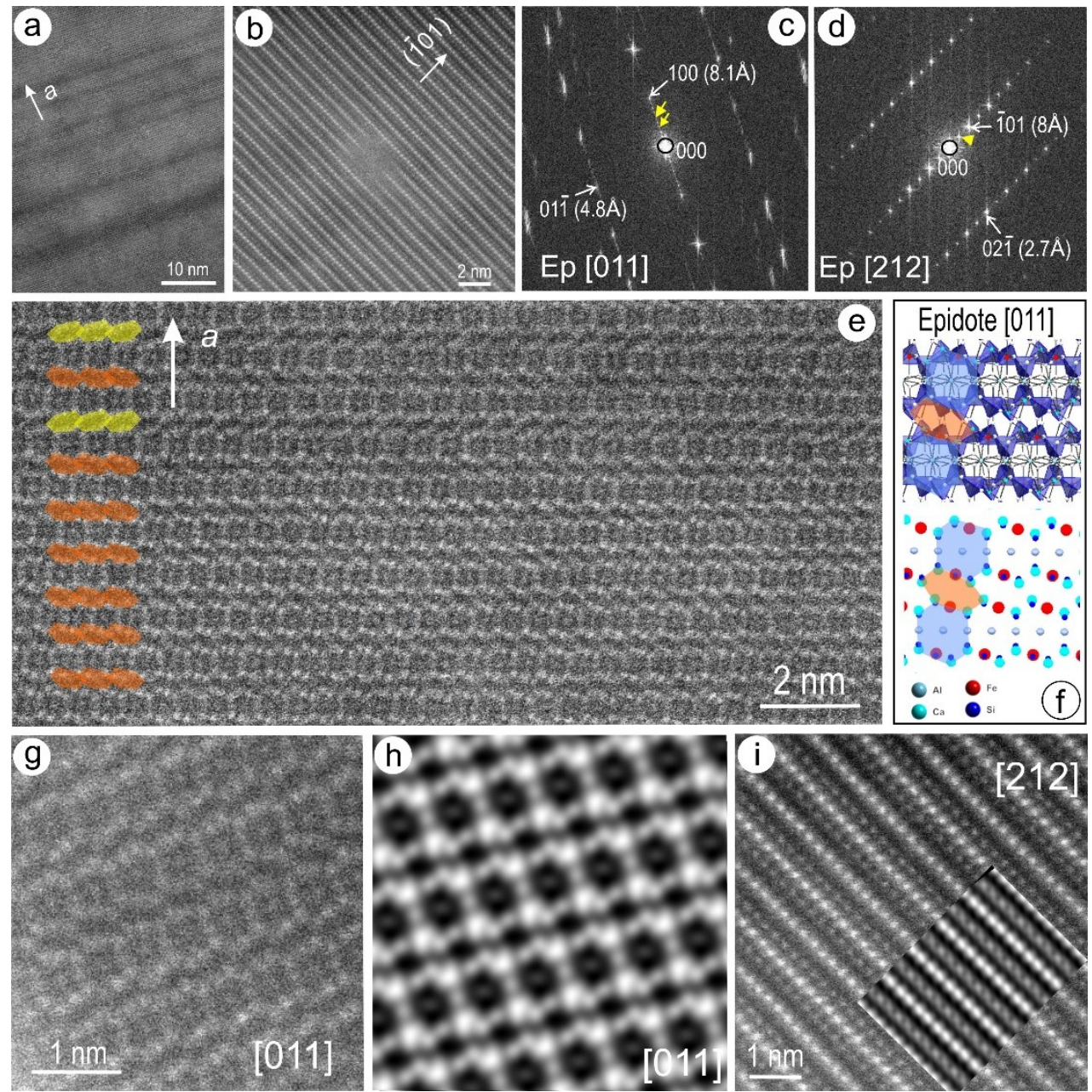

Figure 16. HAADF STEM (a,b) images and FFTs (c,d) showing epidote (Ep) on [011] and [212] zone axes (inclusion in Figure 14h; foil \#4). Note correlation between irregular bands on (a), satellite reflections (arrowed) and streaks along $a^{*}$ indicating stacking disorder. (c) High-resolution image showing the main crystal structural motifs on [011] epidote. (f) Crystal structure models of epidote as polyhedral (up) and atom filling (down) on [011\} zone axis. Note correspondence between ring structure and main motifs on (e). The smaller modules linking the rings are irregularly flipped (yellow and red overlays), indicating disorder on $a$. (g,h) Image and STEM simulation of epidote on [011]. (i) Image and STEM simulation (inset) of epidote on [212] zone axis.

One of the larger rutile patches was also assessed by high-resolution HAADF STEM imaging on [011] zone axis (Supplementary Materials Figure S5). This is relevant for discriminating between ilmenite and rutile by EDS mapping and spot analysis, considering Fe interference from the magnetite host.

\subsection{Magnetite and Si-Fe-Nanoprecipitates}

Magnetite without inclusions was imaged in HAADF STEM mode on three zone axes, tilting the specimen so at least two of these zone axes could be imaged in each foil (Figure 17). In all cases, the studied magnetite represents a single crystal since it shows the same orientation throughout any 
given foil, irrespective of zonation, overprint between magmatic and silician magnetite, or the presence of inclusions. Of the four main zone axes imaged, including for magmatic magnetite in Figure 12d, the $[1 \overline{1} 0]$ and $[11 \overline{2}]$ orientations are better suited for HAADF STEM imaging because they show less dense atom packing on $\{111\}$ directions (Figure 17a-c). Most mineral inclusions imaged are oriented coherently to the host magnetite, as discussed above. Nonetheless, strain induced effects surrounding such mineral inclusions are present in host magnetite as shown by linear defects and nanoscale domains displaying twins and misorientation lattice patterns (Figure 17d-f).
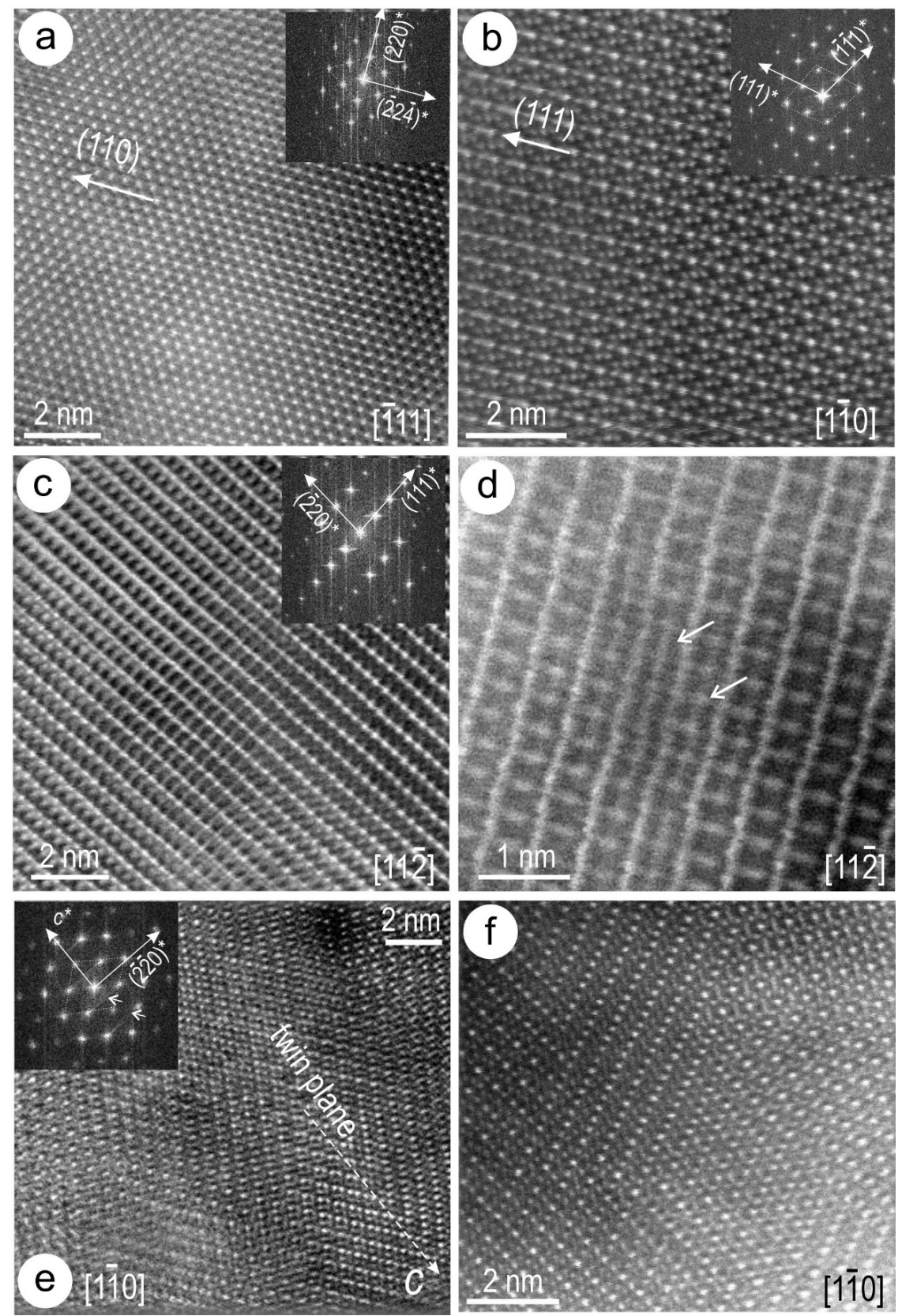

Figure 17. (a-f) High-resolution HAADF STEM images and FFTs (insets) of magnetite on zone axes as marked on each image. Note linear defects in image (d), the micro-twinning in image (e) and nanoscale domains with misorientation in image (f) indicating lattice strain close to inclusions.

The atom distribution on HAADF STEM images for the $[1 \overline{1} 0],[\overline{1} 11]$ and $[11 \overline{2}]$ zone axes in magnetite were assessed by STEM simulation and crystal structure models after calculating the zone 
axis from SAEDs (Figure 18). Such patterns are reproduced in the STEM simulations with good fit on all three zone axes.
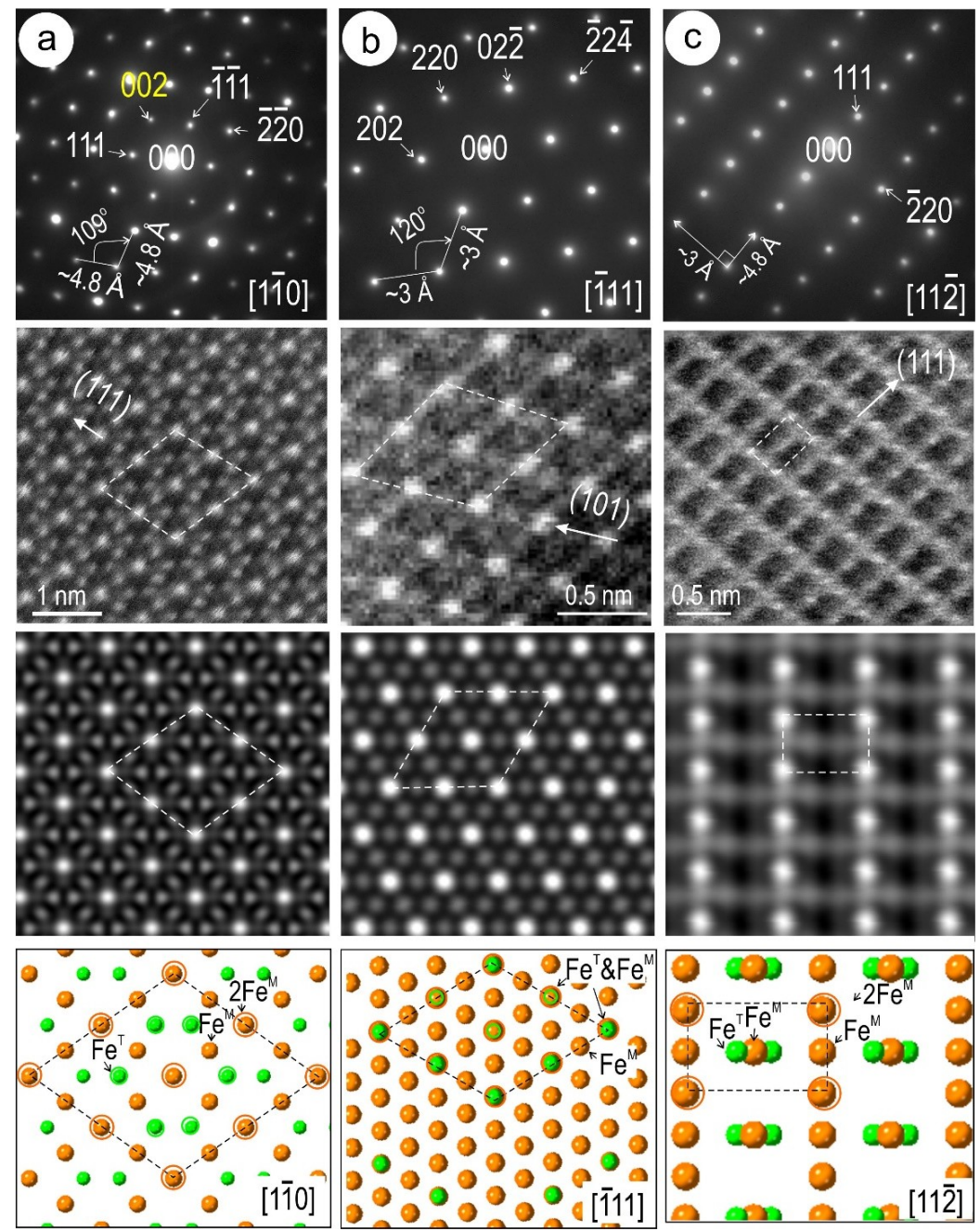

Figure 18. Assessment of HAADF STEM images for magnetite on three main zone axes as marked. Each zone axis is illustrated along the a to c columns representing, from top to bottom: SAED, HAADF image, STEM simulation and crystal model. The presence of forbidden (002) reflections for space group $F d 3 m$, albeit weaker than (004), is observed on SAEDs for [1 $\overline{1} 0]$ zone axis (yellow; a). Such reflections cannot be attributed to double diffraction since they are also present on the FFTs obtained from HAADF STEM images but could be associated with lower symmetry, for example, space group $\bar{F} \overline{4} \mathrm{~m}$ instead of Fd3m (Table 1 ; [3]). Images of magnetite on both $[1 \overline{1} 0]$ and $[\overline{1} 11]$ zone axes display a rhomb-shaped motif with brighter atoms surrounded by 10 and 6 less brighter atoms, respectively $(\mathbf{a}, \mathbf{b})$. The image for $[11 \overline{2}]$ zone axis shows brighter atoms at the corner of a rectangular pattern and also the presence of less bright atoms forming a rectangular subset at half-distance to the main pattern (c). The corresponding crystal structure models show that the bright atoms coincide with positions of higher density atoms along the direction of view, corresponding, in magnetite, to an overlap between two Fe atoms with either the same coordination, or with both types of coordination, tetrahedral (T) and rhombohedral (M). STEM simulation for the $[1 \overline{1} 0]$ image, however, requires a setting of the $F d 3 m$ cell with atom coordinates at origin 1 as given by Yamanaka et al. [16] rather than origin 2, the conventional setting for magnetite and other "2-3"-type spinels (Table 1 ). Simulation using the $\bar{F} \overline{4} 3 m$ space group of lower symmetry does not give such a good fit as $F d 3 m$ cell with atom coordinates at origin 1.

Si-Fe-nanoprecipitates are present throughout all seven foils representing the silician magnetite from Olympic Dam studied here (foils \#1-7; Table 2). These are recognizable as darker inclusions on the 
HAADF STEM images, varying in both size (from a few to some tens of nm) and density. Bleb-shaped inclusions are particularly abundant in the massive magnetite from the northern side of the deposit, whereas a tendency towards, sub-euhedral geometrical-shaped inclusions is observed in silician magnetite from the E transect (Figure 19a-c, Supplementary Materials Figures S6-S9). In all cases the HR imaging shows epitaxial relationships between the host, Si-free magnetite and the Si-bearing, magnetite nanoprecipitate (Figure 19d-f). Silicon is measured on the STEM maps and by EDS spot analysis, albeit with variable peak intensity, and in some cases, other elements $(\mathrm{Ca}, \mathrm{Mg}$, and $\mathrm{K})$ are also measured (spectra in Figure 19 and Supplementary Materials Figures S6-S9). High-resolution imaging of $\mathrm{Si}-\mathrm{Fe}-$ nanoprecipitates occurring as blebs shows the same atomic arrangement as magnetite (compare Figures 18a and 19g), suggesting they both have the same spinel structure. This is also concordant with STEM simulations of Si-magnetite with Fd3m vacancy disorder (Si-magnetite models of Yamanaka et al. [16]; Table 1).
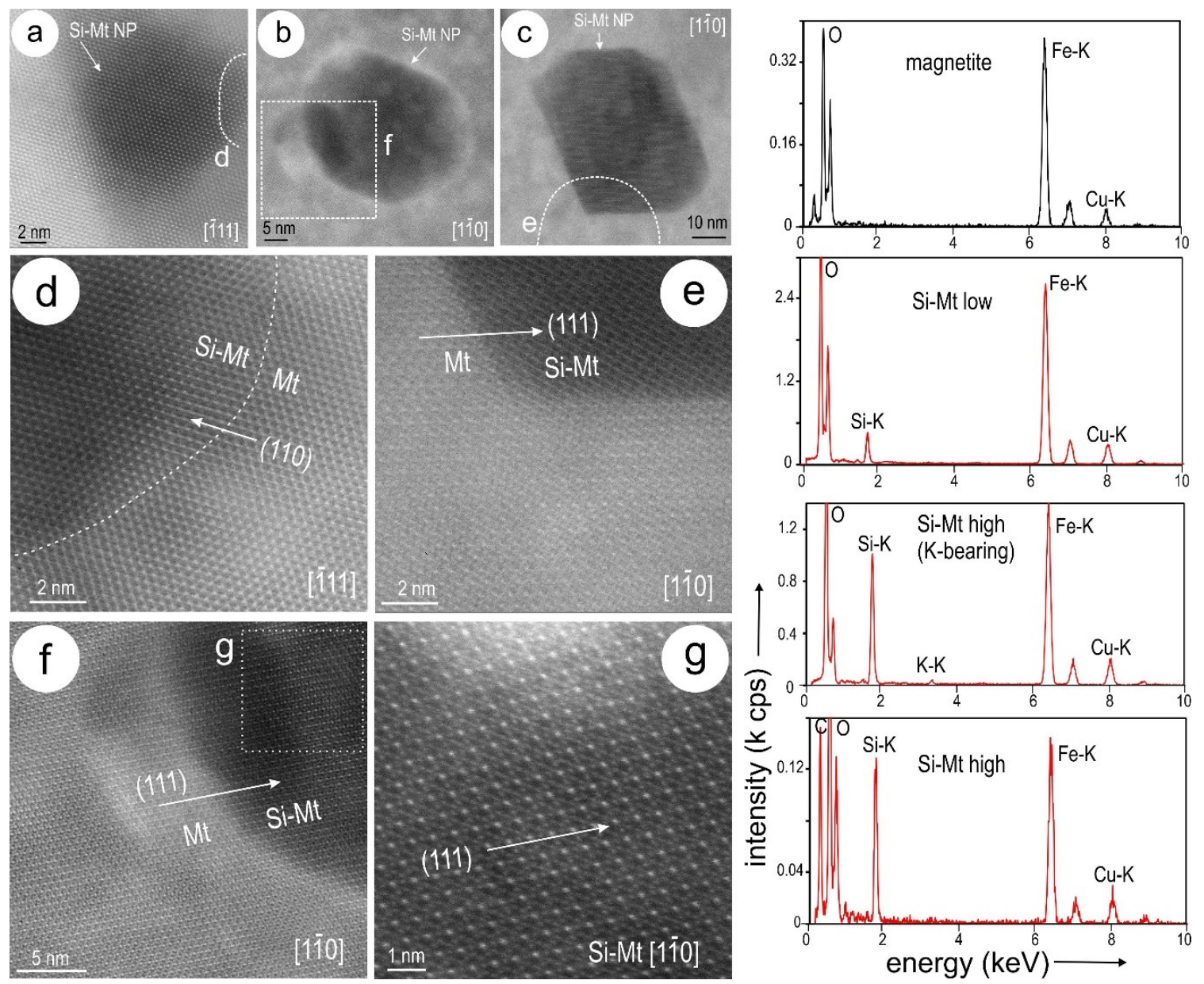

Figure 19. HAADF STEM images (a-g) and EDS STEM spectra (to the right) representing Si-Fe-nanoprecipitates with bleb morphology. (a-c) Variable morphology of bleb precipitates from smallest, rounded NPs in (a) to sub-rounded and short-prismatic in (b,c). (d-f) Details of boundaries between the precipitate and host magnetite (from areas as highlighted in (a)) on zone axes as marked showing epitaxial relationships between the two phases. (g) High-resolution image of the bleb precipitate (from area marked in $\mathbf{f}$ ) showing the same atomic arrangement as magnetite on [1 10$]$ zone axis. Note magnetite outside the blebs is Si-free and the variation between $\mathrm{Si} / \mathrm{Fe}$ ratio from low to high) in spectra to the right. The $\mathrm{Cu}$ signal on the spectra is from the TEM grids.

$\mathrm{Si}-\mathrm{Fe}$-nanoprecipitates with maghemite ordering corresponding to $\gamma-\mathrm{Fe}_{1.5} \mathrm{SiO}_{4}\left(P 4_{3} 32\right.$ symmetry (Table 1) were identified from location \#4 (foil \#5). In this case, the nanoprecipitates are represented by 
narrow strips with widths corresponding to $2 n(n=1,2, \ldots)$ of $d_{111}$ in magnetite. On the HAADF STEM images these appears still darker than the blebs and are stacked along conjugate $d_{111}$ and $d_{11-1}$ directions in magnetite within the coarser, Si-Fe-blebs or directly in the magnetite outside of them (Figure 20a,b). The Si-Fe-nanoprecipitate blebs hosting the darker strips also display sets of twins and show disorder as misorientation nanodomains cutting across the $2 d_{111}$ in the Si-magnetite blebs (Figure 20c,d).
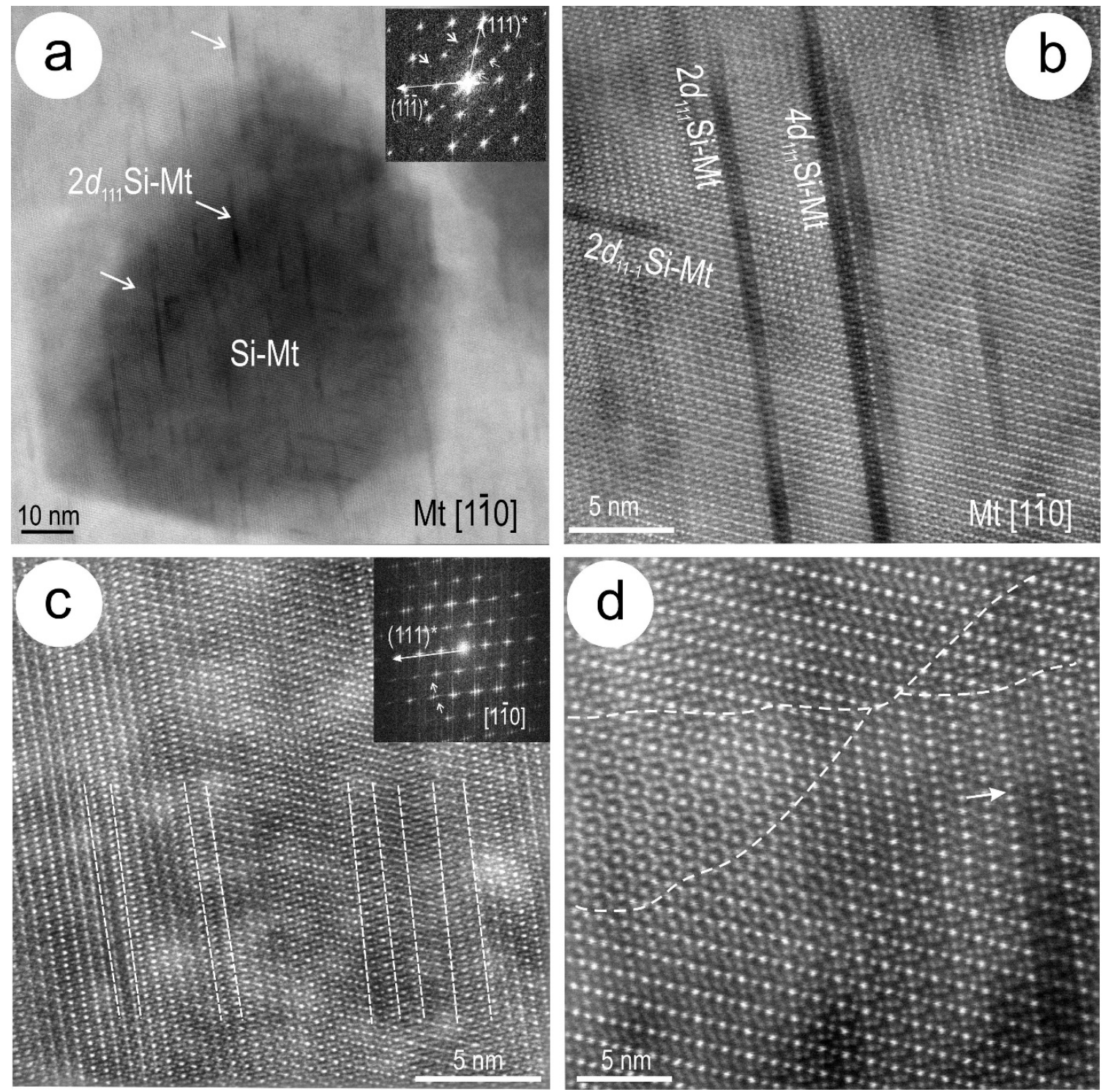

Figure 20. HAADF STEM images showing the two types of Si-Fe-nanoprecipitates (foil \#5) on [1]̄0] zone axis in magnetite (Mt). (a) Overlap between Si-Fe-nanoprecipitates as narrow strips ( $\left.2 \mathrm{~d}_{111} \mathrm{Si}-\mathrm{Mt}\right)$ and blebs (Si-Mt). Satellite reflections on FFT (arrowed, inset) are concordant with interpretation of maghemite superstructure [21]. (b) Strip nanoprecipitates occur along conjugate $d_{111}$ and $d_{11 \overline{1}}$ directions in magnetite with $2 n(n=1,2, \ldots)$ widths. (c,d) Nano-twins and -misorientation domains (dashed line) in the bleb precipitates containing the $2 n . d_{111}$ strips.

Although it is difficult to unequivocally discriminate between magnetite and the Si-Fe-nanoprecipitate structure on the image where they overlap, intensity line profiles show variation attributable to $\mathrm{Si}$ substitution in the tetrahedral site and vacancies in the octahedral site in accordance with the crystal structural formula $\left[\square_{0.5} \mathrm{Fe}^{2+}{ }_{0.5}\right]^{\mathrm{VI}}\left[\mathrm{Fe}^{3+}\right]^{\mathrm{VI}} \mathrm{Si} \mathrm{IV}_{\mathrm{O}_{4}}$ for $\gamma-\mathrm{Fe}_{1.5} \mathrm{SiO}_{4}[29]$ (Figure 21a). Comparison between images (Figure 21b,c) and STEM simulations (Figure 21d,e) for $\gamma-\mathrm{Fe}_{1.5} \mathrm{SiO}_{4}$ show a relatively good fit on $[\overline{1} 11]$ zone axis. The decrease in intensity across the 
Si-Fe-nanoprecipitate strip is somewhat reproduced in the model layering for the [110] zone axis. The fit for the Si-Fe-nanoprecipitate ${ }_{[\overline{1} 11]}$ images implies that some blebs may be also nanoprecipitates with the same vacancy-ordered structure as considered for the $d_{111}$ strips.
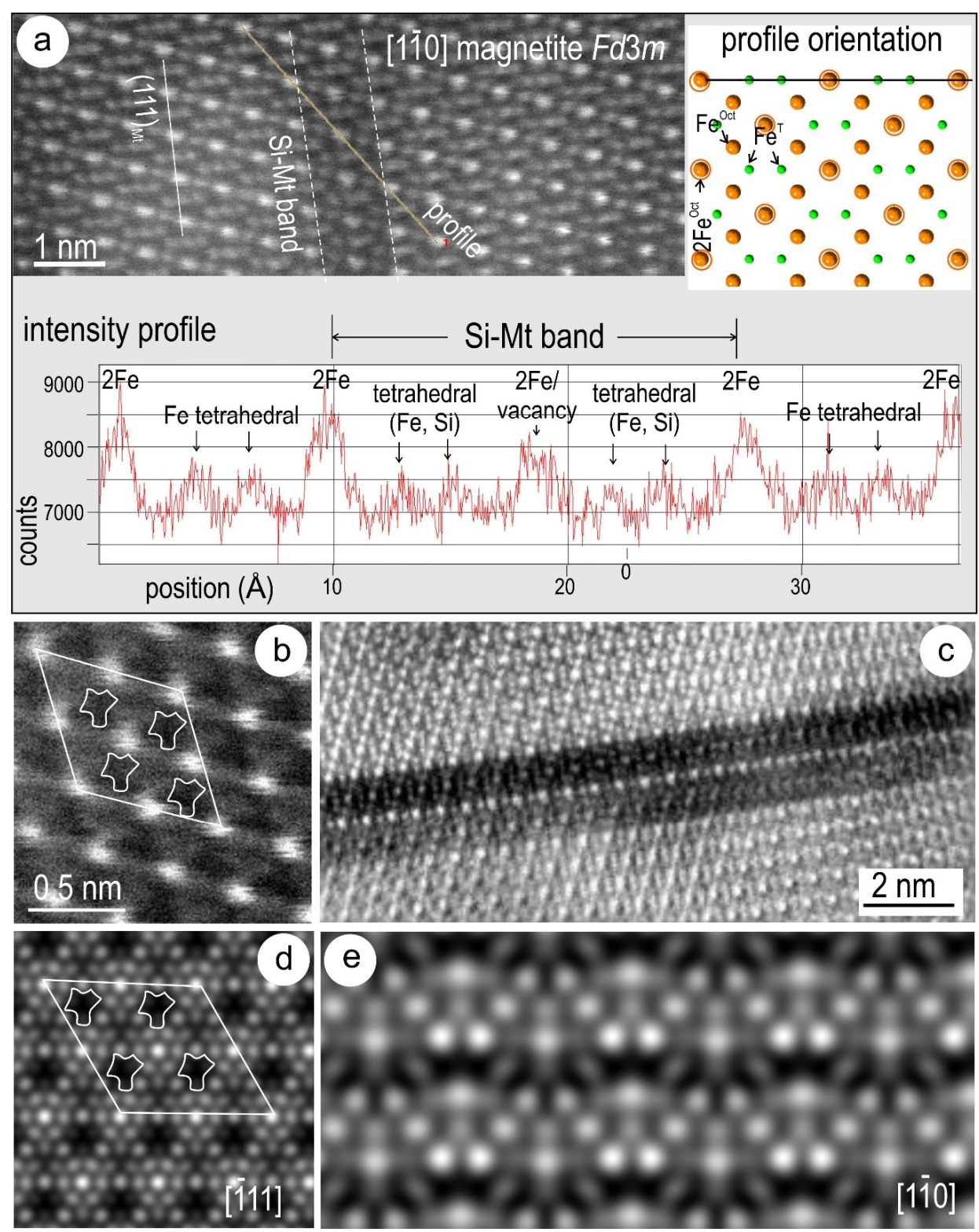

Figure 21. (a) HAADF STEM image (left) and profile (below) along orientation as marked on the crystal model (right) showing variation of HAADF signal intensity across the $2 d_{111}$ nanoprecipitate (Si-Mt) in magnetite (Mt). This is compatible with vacancies in both $\mathrm{T}$ and $\mathrm{M}$ sites as suggested by DFT calculations for $\gamma-\mathrm{Fe}_{1.5} \mathrm{SiO}_{4}$ [29]. (b,c) Detail of $\mathrm{Si}-\mathrm{Mt}$ bleb ${ }_{[\overline{1} 11]}$ and $2 d_{111}$ strip ${ }_{[1 \overline{1} 0]}$ with STEM simulations (using $\gamma-\mathrm{Fe}_{1.5} \mathrm{SiO}_{4}[29]$ ), shown underneath in (d,e) showing a relative good fit.

\section{Discussion}

\subsection{What Is "Silician Magnetite"?}

Two varieties of silician magnetite with cubic structures of different symmetry (spinel- and maghemite-types; Table 1) are documented from Si-Fe-nanoscale inclusions or "nanoprecipitates" in magnetite from Olympic Dam. The spinel-type is, to our knowledge, the first natural occurrence of analogous compounds synthesized at high pressure and T [16]. 
Superposition between the two types of Si-Fe-nanoprecipitates suggests they can be derived from one another, with the maghemite-type most likely formed later via Fe-vacancy ordering. This is suggested by the association between maghemite-type nanoprecipitates along $d_{111}$-magnetite, with twinning and defects in the spinel-type blebs (Figure 20). However, the nm-sized strips of maghemite-type develop pervasively along $\{111\}$ planes in magnetite, indicating that the ordering process is not restricted to the spinel-type blebs but can also occur within the host magnetite. Epitaxial relationships between all nanoprecipitates and host magnetite is supportive of crystallographically-controlled (non-random) growth mechanisms such as exsolution from solid solution, or fluid-mineral precipitation and/or replacement with a sharp reaction interface (coupled dissolution-(re)precipitation reaction; CDRR).

Differences in temperature and/or growth rates can explain the presence of two different types of Si-Fe-nanoprecipitates in the same sample: higher-T and/or faster growth rates for the blebs with spinel structure; and vice versa for the strips with vacancy-ordered lower cubic structure. The wide variation in $\mathrm{Si} / \mathrm{Fe}$ ratios within the $\mathrm{Si}$-magnetite blebs and the association with $\mathrm{SiO}_{2}$ nanoprecipitates along the same growth zone in magnetite (Figure 19 and Supplementary Materials Figures S6-S9) indicates fluctuating conditions during precipitation from fluids containing $\mathrm{Si}$ - and $\mathrm{Fe}$-complexes. In contrast, the maghemite-type $d_{111}{ }^{-}$magnetite nanoprecipitates have a low $\mathrm{Si} / \mathrm{Fe}$ ratio but nonetheless contain measurable $\mathrm{K}$ and $\mathrm{Al}$, elements which are also responsible for the fine-scale, oscillatory banding observed in host magnetite. Such characteristics imply that both types of Si-Fe-nanoprecipitates formed from hydrothermal fluids with subtle variation in chemistry rather than exsolution from a phase with intermediate composition in the magnetite-ahrensite solid-solution. If true, then Si-Fe-nanoprecipitates should also occur in other host minerals, such as hematite, olivine-group minerals or other silicates.

We show that single grains of magnetite which are all $\mathrm{Si}-(\mathrm{Ca}, \mathrm{Mg})$-bearing at the $\mu \mathrm{m}$-scale [37] contain two types of Si-Fe-inclusions, as well as an eclectic range of other nanominerals. By illustrating the discrepancy between minor element distributions with the scale of mapping, from continuous bands to discrete nanomineral inclusions in the same sample, we raise the question of whether the phases considered as silician magnetite in many previous studies might actually feature characteristics comparable to those described here, if analyzed at the nanoscale.

\subsection{Petrogenetic Significance of Silician Magnetite: Si-Fe-Nanoprecipitates and other Mineral Inclusions}

Formation of calc-silicates (actinolite, diopside, epidote) along the same growth zones as the $\mathrm{Si}$-Fe-blebs is concordant with higher temperatures of at least $\sim 400^{\circ} \mathrm{C}$, based on comparison with analogous skarn assemblages [57] and also concordant with the upper stability limit of ferroactinolite $\left(420{ }^{\circ} \mathrm{C}\right.$ at $2 \mathrm{kbar}$; [58]). Calcic amphiboles and clinopyroxenes are present in greater proportions in magnetite-apatite-rich deposits considered kin to IOCGs (subclass iron oxide apatite, IOA) such as the Proterozoic Kiruna deposit, Sweden (e.g., [59]), or younger Cretaceous-Tertiary analogue deposits from the Chilean Andes such as Los Colorados and El Laco [35,60]. Actinolite associated with magnetite is present in significantly sized accumulations, either forming pegmatites (Kiruna; [59]), or as the matrix/groundmass for magnetite (Los Colorados; [35]). In the latter example, the presence of actinolite and several other silicates as nanoparticles within magnetite is interpreted as evidence for a hydrothermal origin [35]. In contrast, a magmatic origin is considered for Kiruna actinolite based on the stability of high-Mg actinolite up to $600-880{ }^{\circ} \mathrm{C}$ at $1-4 \mathrm{kbar}$, concordant with conditions of Fe-P-rich melt formation [59]. Vein diopside (associated with magnetite and anhydrite) at El Laco, the youngest (Pliocene-Pleistocene) known IOA deposit, is attributed to crystallization from iron-rich, hydrosaline melts and distinct from the alkali-calcic hydrothermal alteration in host andesite [60].

At Olympic Dam, (ferro)actinolite and accompanying calc-silicates found as nanomineral inclusions in silician magnetite are distinct from associations characteristic of magmatic magnetite (inclusions of spinels + Fe-Ti-oxides; Figures 9 and 11). Pseudomorphic, CDRR replacement of magmatic magnetite by silician magnetite is typified by abundant rutile (Figure 9), indicating interface-mediated element exchange since the newly formed mineral inherits Ti from precursor magnetite. The data 
here from Olympic Dam contradict the suggestion of Newberry [14] that $\mathrm{SiO}_{2}$ is present in magmatic magnetite from granites since we show a clear difference between magmatic and Si-magnetite from the same granite.

Local alkali-calcic alteration (nanoscale inclusions of epidote in RDG-hosted hydrothermal hyalophane [50,52] from the same location \#5 as the magmatic magnetite studied here) represents the earliest hydrothermal stage at Olympic Dam [37]. If early alteration of magmatic feldspars and breakdown of mafic minerals takes place via CDRR, as has been shown for Olympic Dam [48,51], then fluid-mineral reactions can be arrested on the $\mathrm{cm}$ - to m-scale enabling incorporation into new minerals, including the silician magnetite discussed here. The source of both $\mathrm{Fe}$ and $\mathrm{Si}$ may, however, be the hydrothermal fluids released from the granite at this stage. Formation of early, hydrothermal magnetite was modelled considering $\mathrm{pH}$ variation across the buffer between $\mathrm{K}$-feldspar and sericite [37]. This transition is recorded from Si-magnetite in a sample from location \#4, where a strong overprint of silician magnetite is also observed (e.g., abundant lattice-scale defects such as twinning and faults, $\mathrm{K}$ - and $\mathrm{Al}$-banding, $\mathrm{SiO}_{2}$-rich inclusion subpopulations and replacement by fluorite; Figures 8, 14a and 20). Variable degrees of coarsening of Si-Fe-blebs during interaction with fluids enriched in elements typical of that stage of fluid evolution or local environment could explain the variation in abundance, size and, in particular, variation in the measured contents of $\mathrm{Ca}$ and $\mathrm{Mg}$ in some of the Si-Fe-blebs. The presence of a diverse range of other inclusions (e.g., carbonates, chlorite or sphalerite) points at local mineralogical/geochemical differences between the studied locations. The sample from the Deep Mineralization (location \#3) is the most varied in terms of mineralogy and is the only case where epidote has been identified in the silician magnetite. Availability of $\mathrm{Al}$ necessary to form epidote may be achieved by direct replacement of plagioclase by Fe-oxides, as has been reported from the same sample, at the contact between RDG and a felsic volcanic, containing silician magnetite studied here [37].

Although nanomineral inclusions reported in "hydrothermal" magnetite from Los Colorados [35] comprise actinolite and diopside like those described from Olympic Dam, they differ in the abundance and speciation of Fe-Ti-minerals (Ti-rich magnetite and ulvöspinel) and by abundant phlogopite. The $\mu \mathrm{m}$-scale distribution of minor elements in Los Colorados magnetite was mapped as oscillatory zoning, in which Ti-rich bands alternate with bands containing high $\mathrm{Al}, \mathrm{Si}, \mathrm{Ca}$ and $\mathrm{Mg}$. Therefore, the nature of nanomineral inclusions in magnetite may offer a discrimination between IOCGs sensu stricto and IOA deposits. The occurrence of calc-silicate inclusions in silician magnetite, comparable to those discussed here for IOCG deposits, are predictable for silician magnetite in skarns where this is widely reported (e.g., [33]). Studies of magnetite across micron- to nanoscales of observation could be used to discriminate prograde versus retrograde stages in skarns.

A BIF-like scenario for silician magnetite formation [24] can explain the entrapment of nanoparticles containing U-, W-(Mo), Y-As, or As-S within Si-Fe-blebs, which is recorded only in the massive magnetite from Olympic Dam (location \#1). If this magnetite is inherited from Fe-rich horizons within a sedimentary rock (Wallaroo Group?) that predates the host RDG (U-Pb age of $1.75 \mathrm{Ga}$ for coarse magnetite [56]), then the presence of $U$ dissolved in the same pore fluids supplying aqueous silica and Fe could explain the observation of U-bearing nanoparticles attached to Si-Fe-blebs along the crystal zoning. Alternatively, such U-bearing nanoparticles (or W-Mo-bearing nanoparticles) can be attributed to the IOCG overprint, particularly when these are observed along trails crosscutting the crystal zoning in smaller grains. The presence of both Y-arsenates (chernovite) and sulphoarsenides (arsenopyrite) indicates locally buffered reactions indicative of CDRR during this overprinting event. Sulphidation of Si-magnetite during precipitation of sulphides (chalcopyrite + pyrite) typical of the outer shell at Olympic Dam [37] is further evidence for considering that an IOCG overprint is recorded by the silician magnetite. The presence of abundant inclusions of siderite in magnetite inferred by Ciobanu et al. [36] cannot be confirmed from the present data, although carbonates are present along the bands with Si-Fe-blebs. Such a discrepancy highlights the importance of advanced electron microscopy in studying ore minerals at appropriate scales of observation and using complementary techniques [52,61]. 


\section{Conclusions and Implications}

Silician magnetite containing Si-Fe-nanoprecipitates and an eclectic range of nanomineral inclusions (ranging in size from fine particles to nanoparticles) is typical of the weakly-mineralized granite forming the outer shell around the Olympic Dam deposit. This is the first report of Si-magnetite nanoprecipitates with spinel-type structure and the second occurrence of $\gamma-\mathrm{Fe}_{1.5} \mathrm{SiO}_{4}$ phase with maghemite structure. The nanoprecipitates have distinct morphologies, occurring as bleb-like and nm-wide strips along $d_{111}$ in magnetite, respectively. All inclusions are epitaxial with magnetite and contribute to crystal zoning with respect to $\mathrm{Si}+(\mathrm{Ca}, \mathrm{Mg})$ at the $\mu \mathrm{m}$-scale.

Calc-silicate inclusions, comprising (ferro)actinolite, diopside and epidote, are identified from STEM-EDS analysis combined with atom-scale resolution HAADF STEM imaging and STEM simulations. Such mineral associations, along with compositional zoning in the magnetite with respect to $\mathrm{K}$ and $\mathrm{Al}$, characterize early, alkali-calcic alteration in the IOCG system at Olympic Dam.

Magmatic magnetite from host granite is characterized by nanomineral associations of hercynite-ulvöspinel-ilmenite that are distinct from those present in the Si-magnetite. An increase in the abundance of rutile inclusions in Si-magnetite formed by pseudomorphic replacement of magmatic magnetite represents further evidence for metasomatic reactions at this stage via a coupling of dissolution with (re)precipitation rates.

Uranium-, W-(Mo), Y-As- and As-S-nanoparticles attached to Si-Fe-nanoprecipitates are typical of magnetite from a massive interval previously considered to represent blocks inherited from an older BIF protolith. The increase in abundance of such nanoparticles along trails that conspicuously crosscut crystal zoning is evidence for the IOCG overprint.

Overprinting of Si-magnetite during transition from K-feldspar to sericite is also expressed as abundant lattice-scale defects (twinning, faults) associated with the transformation of nanoprecipitates with spinel structure into maghemite via Fe-vacancy ordering.

Silician magnetite has petrogenetic value in defining stages of ore deposit evolution at Olympic Dam and for IOCG systems elsewhere. The new data also add new perspectives into the definition of silician magnetite and its occurrence in ore deposits.

Supplementary Materials: The following are available online at http://www.mdpi.com/2075-163X/9/5/311/s1, Figure S1: HAADF STEM images showing the 8 foils (Table 1) analyzed in this study and the location of element maps as marked.; Figure S2: HAADF STEM image, EDS maps and profile showing calc-silicate inclusions in magnetite (foil \#4); Figure S3: HAADF STEM image, EDS maps and profile showing Al- and K-bearing Si-Fe-nanoprecipitates (Si-Mt) in magnetite (foil \#5); Figure S4: HAADF STEM image, EDS maps and spectrum showing (ferro)actinolite inclusion in magnetite (foil \#4); Figure S5: HAADF STEM images (a, c, d), spectrum (b) and FFT (e) showing rutile (Rt) on [0-11] zone axis (foil \#4). Figure S6: HAADF STEM images and EDS spectra showing variation in composition of Si-Fe-blebs ( $\mathrm{Si}-\mathrm{Mt}$ ) from coarse magnetite (Mt) (foil \#1); Figure S7: HAADF STEM images showing textures of silician magnetite of medium-sized grains (foil \#2); Figure S8: HAADF STEM images and EDS spectra showing variation in composition of $\mathrm{Si}-\mathrm{Fe}-\mathrm{blebs}(\mathrm{Si}-\mathrm{Mt})$ along crystal zoning in medium-sized magnetite (Mt) (foil \#2); Figure S9. HAADF STEM images and EDS spectra showing variation in composition of Si-Fe-blebs (Si-Mt) along trails in medium-sized magnetite (Mt) (foil \#2).

Author Contributions: C.L.C. conceived and coordinated this contribution. Analytical work was carried out by C.L.C. M.R.V.-I., and A.S. (operating Titan Themis) assisted by L.C.-D. and B.P.W. K.E. supplied samples, guidance and insight. The paper was written by C.L.C. and N.J.C., with contributions from M.R.V.-I., L.C.-D., K.E., B.P.W. and A.S.

Funding: C.L.C., L.C.D. and M.R.V.-I. acknowledge support from the "FOX" project (Characterization of Fe-oxides), supported by BHP Olympic Dam and the South Australian Government Mining and Petroleum Services Centre of Excellence. N.J.C. acknowledges additional support from the ARC Research Hub for Australian Copper-Uranium (Grant IH130200033).

Acknowledgments: Additional analytical support from staff at Adelaide Microscopy is appreciated. We gratefully acknowledge the constructive comments of three journal reviewers.

Conflicts of Interest: The authors declare no conflict of interest. 


\section{References}

1. Biagioni, C.; Pasero, M. The systematics of the spinel-type minerals: An overview. Am. Mineral. 2014, 99, $1254-1264$. [CrossRef]

2. Hill, R.J.; Craig, J.R.; Gibbs, G.V. Systematics of the spinel structure type. Phys. Chem. Miner. 1979, 4, 317-339. [CrossRef]

3. Fleet, M.E. The structure of magnetite: Symmetry of cubic spinels. J. Solid State Chem. 1986, 62, 75-82. [CrossRef]

4. Verwey, E.J.W.; de Boer, J.H. Cation arrangement in a few oxides with crystal structures of the spinel type. Recueil des Travaux Chimiques des Pays Bas 1936, 55, 531-540. [CrossRef]

5. Fleet, M.E. The structure of magnetite. Acta Cryst. B 1981, 37, 917-920. [CrossRef]

6. Ramdohr, P. The Ore Minerals and Their Intergrowths; English Translation of the 3rd Edition; Pergamon Press: Oxford, UK, 1969; 1174p.

7. Bowles, J.F.W.; Howie, R.A.; Vaughan, D.J.; Zussman, J. Rock-forming Minerals: Non-Silicates: Oxides, Hydroxides and Sulphides; The Geological Society of London: London, UK, 2011; 920p.

8. Buddington, A.F.; Lindsley, D.H. Iron-titanium oxide minerals and synthetic equivalents. J. Petrol. 1964, 5, 310-357. [CrossRef]

9. Haggerty, S.E. Apollo 14: Subsolidus reduction and compositional variations of spinels. In Proceedings of the 3rd Lunar Science Conference; MIT Press: Cambridge, MA, USA, 1972; Volume 1, pp. 305-332.

10. Haselton, J.C.; Nash, W.P. Ilmenite-orthopyroxene intergrowths from the moon and the Skaergaard intrusion. Earth Plan. Sci. Lett. 1975, 26, 287-291. [CrossRef]

11. Mücke, A. Magnetite, ilmenite and ulvite in rocks and ore deposits: Petrography, microprobe analyses and genetic implications. Mineral. Petrol. 2003, 77, 215-234. [CrossRef]

12. Gao, W.; Ciobanu, C.L.; Cook, N.J.; Slattery, A.; Huang, F.; Wang, D. Nanoscale study of lamellar exsolutions in clinopyroxene from olivine gabbro: Recording crystallization sequences in iron-rich layered intrusions. Am. Mineral. 2019, 104, 244-261.

13. Ma, C.; Tschauner, O.; Beckett, J.R.; Liu, Y.; Rossman, G.R.; Sinogeikin, S.V.; Smith, J.S.; Taylor, L.A. Ahrensite, $\gamma-\mathrm{Fe}_{2} \mathrm{SiO}_{4}$, a new shock-metamorphic mineral from the Tissint meteorite: Implications for the Tissint shock event on Mars. Geochim. Cosmochim. Acta 2016, 184, 240-256. [CrossRef]

14. Newberry, N.G.; Peacor, D.R.; Essene, E.J.; Geissman, J.W. Silicon in Magnetite: High-resolution microanalysis of magnetite-ilmenite intergrowths. Contrib. Mineral. Petrol. 1982, 80, 334-340. [CrossRef]

15. Ohtaka, O.; Tobe, H.; Yamanaka, T. Phase equilibria for the $\mathrm{Fe}_{2} \mathrm{SiO}_{4}-\mathrm{Fe}_{3} \mathrm{O}_{4}$ system under high pressure. Phys. Chem. Miner. 1997, 24, 555-560. [CrossRef]

16. Yamanaka, T.; Shimazu, H.; Ota, K. Electric conductivity of $\mathrm{Fe}_{2} \mathrm{SiO}_{4}-\mathrm{Fe}_{3} \mathrm{O}_{4}$ spinel solid solutions. Phys. Chem. Miner. 2001, 28, 110-118. [CrossRef]

17. Shcheka, S.A.; Romanenko, I.M.; Chubarov, V.M.; Kurentsova, N.A. Silica-bearing magnetites. Contrib. Mineral. Petrol. 1977, 63, 103-111. [CrossRef]

18. Shiga, Y. Silician magnetite from the Kamaishi Mine, Japan. Min. Geol. 1988, 38, 437-440.

19. Huberty, J.M.; Konishi, H.; Heck, P.R.; Fournelle, J.H.; Valley, J.W.; Xu, H. Silician magnetite from the Dales Gorge member of the Brockman iron formation, Hamersley Group, Western Australia. Am. Mineral. 2012, 97, $26-37$. [CrossRef]

20. Shimazaki, H. On the occurrence of silician magnetites. Resour. Geol. 1998, 48, 23-29. [CrossRef]

21. Grau-Crespo, R.; Al-Baitai, A.Y.; Saadoune, I.; De Leeuw, N.H. Vacancy ordering and electronic structure of $\gamma-\mathrm{Fe}_{2} \mathrm{O}_{3}$ (maghemite): A theoretical investigation. J. Phys. Condens. Matter 2010, 22, 255401. [CrossRef]

22. Fleet, M.E. The structure of magnetite: Two annealed natural magnetites, $\mathrm{Fe}_{3.005} \mathrm{O}_{4}$ and $\mathrm{Fe}_{2.96} \mathrm{Mg}_{0.04} \mathrm{O}_{4}$. Acta Cryst. 1984, C40, 1491-1493. [CrossRef]

23. Lenaz, D.; Skogby, H. Structural changes in the $\mathrm{FeAl}_{2} \mathrm{O}_{4}-\mathrm{FeCr}_{2} \mathrm{O}_{4}$ solid solution series and their consequences on natural Cr-bearing spinels. Phys. Chem. Miner. 2013, 40, 587-595. [CrossRef]

24. Ardit, M.; Cruciani, G.; Dondi, M. Structural relaxation in tetrahedrally coordinated $\mathrm{Co}^{2+}$ along the gahnite-Co-aluminate spinel solid solution. Am. Mineral. 2012, 97, 1394-1401. [CrossRef]

25. Pavese, A.; Levy, D.; Hoser, A. Cation distribution in synthetic zinc ferrite $\left(\mathrm{Zn}_{0.97} \mathrm{Fe}_{2.02} \mathrm{O}_{4}\right)$ from in situ high temperature neutron powder diffraction Sample at T $=300$ K. Am. Mineral. 2000, 85, 1497-1502. [CrossRef] 
26. Yagi, T.; Marumo, F.; Akimoto, S.I. Crystal structures of spinel polymorphs of $\mathrm{Fe}_{2} \mathrm{SiO}_{4}$ and $\mathrm{Ni}_{2} \mathrm{SiO}_{4}$. Am. Mineral. 1974, 59, 486-490.

27. Hazen, R.M.; Downs, R.T.; Finger, L.W. Crystal chemistry of ferromagnesian silicate spinels: Evidence for Mg-Si disorder. Am. Mineral. 1993, 78, 1320-1323.

28. Bosi, F.; Halenius, U.; Skogby, H. Crystal chemistry of the magnetite-ulvöspinel series. Am. Mineral. 2009, 94, 181-189. [CrossRef]

29. Xu, H.-F.; Shen, Z.-Z.; Konishi, H. Si-magnetite nano-precipitates in silician magnetite from banded iron formation: Z-contrast imaging and ab initio study. Am. Mineral. 2014, 99, 2196-2202. [CrossRef]

30. Bosi, F.; Biagioni, C.; Pasero, M. Nomenclature and classification of the spinel supergroup. Eur. J. Mineral. 2019, 31, 183-192. [CrossRef]

31. Pecharromán, C.; González-Carreño, T.; Iglesias, J.E. The infrared dielectric properties of maghemite, $\gamma-\mathrm{Fe}_{2} \mathrm{O}_{3}$, from reflectance measurement on pressed powders. Phys. Chem. Miner. 1995, 22, 21-29. [CrossRef]

32. Fujino, K.; Sasaki, S.; Takéuchi, Y.; Sadanaga, R. X-ray determination of electron distributions in forsterite, fayalite and tephroite. Acta Cryst. B 1981, 37, 513-518. [CrossRef]

33. Xu, H.F.; Shen, Z.Z.; Konishi, H.; Fu, P.; Szlufarska, I. Crystal structures of laihunite and intermediate phases between laihunite-1M and fayalite; Z-contrast imaging and ab initio study. Am. Mineral. 2014, 99, 881-889. [CrossRef]

34. Westendorp, R.W.; Watkinson, D.H. Silicon-bearing zoned magnetite crystals and the evolution of hydrothermal fluids at the Ansil Cu-Zn mine, Rouyn-Noranda, Quebec. Econ. Geol. 1991, 86, 1110-1114. [CrossRef]

35. Deditius, A.P.; Reich, M.; Simon, A.C.; Suvorova, A.; Knipping, J.; Roberts, M.P.; Rubanov, S.; Dodd, A.; Saunders, M. Nanogeochemistry of hydrothermal magnetite. Contrib. Mineral. Petrol. 2018, 173, 46. [CrossRef]

36. Ciobanu, C.L.; Cook, N.J.; Ehrig, K.; Wade, B.P.; Kamenetsky, V.S. Trace element signatures in iron oxides from the Olympic Dam IOCG deposit, South Australia. In Proceedings of the Mineral Resources in a Sustainable World, 13th Biennial SGA Meeting, Nancy, France, 24-27 August 2015; Volume 3, pp. 1071-1074.

37. Verdugo-Ihl, M.R.; Ciobanu, C.L.; Cook, N.J.; Ehrig, K.; Courtney-Davies, L. Defining early stages of IOCG systems: Evidence from iron-oxides in the outer shell of the Olympic Dam deposit, South Australia. Miner. Deposita 2019. [CrossRef]

38. Ciobanu, C.L.; Verdugo-Ihl, M.R.; Slattery, A.; Cook, N.J.; Ehrig, K.J.; Courtney-Davies, L. Nanoscale study of silician magnetite from IOCG systems: Examples from the Olympic Dam District (South Australia). In Proceedings of the Quadrennial IAGOD Symposium, Salta, Argentina, 28-31 August 2018; pp. 89-90.

39. Otake, T.; Wesolowski, D.J.; Anovitz, L.M.; Allard, L.F.; Ohmoto, H. Experimental evidence for non-redox transformations between magnetite and hematite under $\mathrm{H}_{2} \mathrm{O}$-rich hydrothermal conditions. Earth Plan. Sci. Lett. 2007, 257, 60-70. [CrossRef]

40. Otake, T.; Wesolowski, D.J.; Anovitz, L.M.; Allard, L.F.; Ohmoto, H. Mechanisms of iron oxide transformations in hydrothermal systems. Geochim. Cosmochim. Acta 2010, 74, 6141-6156. [CrossRef]

41. Barton, M.D. Iron oxide (-Cu-Au-REE-P-Ag-U-Co) systems. In Treatise on Geochemistry, 2nd ed.; Elsevier: Amsterdam, The Netherlands, 2014; Volume 13, pp. 515-541.

42. Ehrig, K.; McPhie, J.; Kamenetsky, V.S. Geology and mineralogical zonation of the Olympic Dam iron oxide $\mathrm{Cu}-\mathrm{U}-\mathrm{Au}-\mathrm{Ag}$ deposit, South Australia. In Geology and Genesis of Major Copper Deposits and Districts of the World, a Tribute to Richard Sillitoe; Hedenquist, J.W., Harris, M., Camus, F., Eds.; Society of Economic Geologists: Littleton, CO, USA, 2012; Volume 16, pp. 237-268.

43. Allen, S.R.; McPhie, J.; Ferris, G.; Simpson, C. Evolution and architecture of a large felsic Igneous Province in western Laurentia: The 1.6 Ga Gawler Range Volcanics, South Australia. J. Volcanol. Geotherm. Res. 2008, 172, $132-147$. [CrossRef]

44. Groves, D.I.; Bierlein, F.P.; Meinert, L.D.; Hitzman, M.W. Iron oxide copper-gold (IOCG) deposits through earth history: Implications for origin, lithospheric setting, and distinction from other epigenetic iron oxide deposits. Econ. Geol. 2010, 105, 641-654. [CrossRef]

45. Cherry, A.R.; Ehrig, K.; Kamenetsky, V.S.; McPhie, J.; Crowley, J.L.; Kamenetsky, M.B. Precise geochronological constraints on the origin, setting and incorporation of ca. $1.59 \mathrm{Ga}$ surficial facies into the Olympic Dam Breccia Complex, South Australia. Precambr. Res. 2018, 315, 162-178. [CrossRef] 
46. Courtney-Davies, L.; Tapster, S.R.; Ciobanu, C.L.; Cook, N.J.; Verdugo-Ihl, M.R.; Ehrig, K.J.; Kennedy, A.K.; Gilbert, S.E.; Condon, D.J.; Wade, B.P. A multi-technique evaluation of hydrothermal hematite UePb isotope systematics: Implications for ore deposit geochronology. Chem. Geol. 2019, 513, 54-72. [CrossRef]

47. Creaser, R.A. Petrogenesis of a Mesoproterozoic quartz latite-granitoid suite from the Roxby Downs area, South Australia. Precambr. Res. 1996, 79, 371-394. [CrossRef]

48. Kontonikas-Charos, A.; Ciobanu, C.L.; Cook, N.J.; Ehrig, K.; Krneta, S.; Kamenetsky, V.S. Feldspar evolution in the Roxby Downs Granite, host to Fe-oxide $\mathrm{Cu}-\mathrm{Au}-(\mathrm{U})$ mineralisation at Olympic Dam, South Australia. Ore Geol. Rev. 2017, 80, 838-859. [CrossRef]

49. South Australian Resources Information Gateway (SARIG) Database. Available online: https://map.sarig.sa. gov.au/ (accessed on 17 February 2019).

50. Kontonikas-Charos, A.; Ciobanu, C.L.; Cook, N.J.; Ehrig, K.; Ismail, R.; Krneta, S.; Basak, A. Feldspar mineralogy and rare-earth element (re)mobilization in iron-oxide copper gold systems from South Australia: A nanoscale study. Mineral. Mag. 2018, 82, S173-S197. [CrossRef]

51. Kontonikas-Charos, A.; Ciobanu, C.L.; Cook, N.J.; Ehrig, K.; Krneta, S.; Kamenetsky, V.S. Rare earth element geochemistry of feldspars: Examples from Fe-oxide $\mathrm{Cu}-\mathrm{Au}$ systems in the Olympic $\mathrm{Cu}-\mathrm{Au}$ Province, South Australia. Mineral. Petrol. 2017, 112, 145-172. [CrossRef]

52. Cook, N.J.; Ciobanu, C.L.; Ehrig, K.; Slattery, A.; Verdugo-Ihl, M.R.; Courtney-Davies, L.; Gao, W. Advances and Opportunities in Ore Mineralogy. Minerals 2017, 7, 233. [CrossRef]

53. Mauger, A.J.; Ehrig, K.; Kontonikas-Charos, A.; Ciobanu, C.L.; Cook, N.J.; Kamenetsky, V.S. Alteration at the Olympic Dam IOCG-U deposit: Insights into distal to proximal feldspar and phyllosilicate chemistry from infrared reflectance spectroscopy. Aust. J. Earth Sci. 2016, 63, 959-972.

54. Verdugo-Ihl, M.R.; Ciobanu, C.L.; Cook, N.J.; Ehrig, K.; Courtney-Davies, L.; Gilbert, S. Textures and U-W-Sn-Mo signatures in hematite from the Cu-U-Au-Ag orebody at Olympic Dam, South Australia: Defining the archetype for IOCG deposits. Ore Geol. Rev. 2017, 91, 173-195. [CrossRef]

55. Dmitrijeva, M.; Ehrig, K.; Ciobanu, C.L.; Cook, N.J.; Verdugo-Ihl, M.R.; Metcalfe, A. Defining IOCG signatures through compositional data analysis: A case study of lithogeochemical zoning from the Olympic Dam deposit, South Australia. Ore Geol. Rev. 2019, 105, 86-101. [CrossRef]

56. Ciobanu, C.L.; Cook, N.J.; Wade, B.P.; Ehrig, K. Ore Minerals Down to Nanoscale: Petrogenetic Implications. Acta Geol. Sin. Engl. Ed. 2014, 88, 1441-1443. [CrossRef]

57. Meinert, L.D.; Dipple, G.M.; Nicolescu, S. World Skarn Deposits. Econ. Geol. 2005, 100, 299-336.

58. Jenkins, D.M.; Bozhilov, K.N. Stability and thermodynamic properties of ferro-actinolite: A re-investigation. Am. J. Sci. 2003, 303, 723-752. [CrossRef]

59. Lledo, H.L.; Jenkins, D.M. Experimental investigation of the upper thermal stability of Mg-rich actinolite; Implications for Kiruna-type iron deposits. J. Petrol. 2008, 49, 225-238. [CrossRef]

60. Tornos, F.; Velasco, F.; Hanchar, J.M. Iron-rich melts, magmatic magnetite, and superheated hydrothermal systems: The El Laco deposit, Chile. Geology 2016, 44, 427-430. [CrossRef]

61. Ciobanu, C.L.; Cook, N.J.; Maunders, C.; Wade, B.P.; Ehrig, K. Focused Ion Beam and Advanced Electron Microscopy for Minerals: Insights and Outlook from Bismuth Sulphosalts. Minerals 2016, 6, 112. [CrossRef]

(C) 2019 by the authors. Licensee MDPI, Basel, Switzerland. This article is an open access article distributed under the terms and conditions of the Creative Commons Attribution (CC BY) license (http://creativecommons.org/licenses/by/4.0/). 